\title{
Planar Graphs Have 1-string Representations
}

\author{
Jérémie Chalopin • Daniel Gonçalves • \\ Pascal Ochem
}

Received: 9 August 2008 / Revised: 15 May 2009 / Accepted: 18 May 2009 /

Published online: 29 July 2009

(C) Springer Science+Business Media, LLC 2009

\begin{abstract}
We prove that every planar graph is an intersection graph of strings in the plane such that any two strings intersect at most once.
\end{abstract}

Keywords Planar graphs $\cdot$ Strings

\section{Introduction}

A string $\mathbf{S}$ is a curve of the plane homeomorphic to a segment. A string $\mathbf{s}$ has two ends, the points of $\mathbf{s}$ that are not ends of $\mathbf{s}$ are internal points of $\mathbf{s}$. Two strings $\mathbf{s}_{\mathbf{1}}$ and $\mathbf{s}_{\mathbf{2}}$ cross if they have a common point $\mathbf{p} \in \mathbf{s}_{\mathbf{1}} \cap \mathbf{s}_{\mathbf{2}}$ and if going around $\mathbf{p}$, we successively meet $\mathbf{s}_{\mathbf{1}}, \mathbf{s}_{\mathbf{2}}, \mathbf{s}_{\mathbf{1}}$, and $\mathbf{s}_{\mathbf{2}}$. This means that a tangent point is not a "crossing." In the following we consider string sets without tangent points.

In this paper, we consider intersection models for simple planar graphs (i.e., planar graphs without loops or multiple edges). A string representation of a graph $G=$ $(V, E)$ is a set $\Sigma$ of strings in the plane such that every vertex $v \in V$ maps to a string $\mathbf{v} \in \Sigma$ and such that $u v \in E$ if and only if the strings $\mathbf{u}$ and $\mathbf{v}$ cross (at least once). Similarly, a segment representation of a graph $G$ is a string representation of $G$ in which the strings are segments.

An abstract of this paper appeared in the Proceedings of the eighteenth annual ACM-SIAM Symposium on Discrete algorithms (SODA 2007).

\footnotetext{
J. Chalopin

LIF, CNRS et Aix-Marseille Université, CMI, 39 rue Joliot-Curie, 13453 Marseille Cedex 13, France

D. Gonçalves (凶)

LIRMM, CNRS et Université Montpellier 2, 161 rue Ada, 34392 Montpellier Cedex 05, France e-mail: daniel.goncalves@lirmm.fr

P. Ochem

LRI, CNRS et Université Paris-Sud, Bât 490, 91405 Orsay Cedex, France
} 
These notions were introduced by Ehrlich et al. [3], who proved the following:

\section{Theorem 1 [3] Planar graphs have a string representation.}

In [9], Koebe proved that planar graphs are the contact graphs of disks in the plane. Note that in this model the curves bounding two adjacent disks are tangent. However by inflating these circles we obtain string representations for planar graphs. In his $\mathrm{PhD}$ thesis, Scheinerman [10] conjectures a stronger result:

\section{Conjecture 1 [10] Planar graphs have a segment representation.}

Hartman et al. [8] and de Fraysseix et al. [4] proved Conjecture 1 for bipartite planar graphs. Castro et al. [1] proved Conjecture 1 for triangle-free planar graphs. Recently de Fraysseix and Ossona de Mendez [6] extended this to planar graphs that have a 4-coloring in which every induced cycle of length 4 uses at most three colors. Observe that, since parallel segments never cross, a set of parallel segments in a segment representation of a graph induces a stable set of vertices. The construction in $[4,8]$ (resp. [1]) has the nice property that there are only two (resp. three) possible slopes for the segments. So the construction induces a 2-coloring (resp. 3-coloring) of $G$. Note that Castro et al. do not prove the 3-colorability of triangle-free planar graphs, they use such coloring of the graphs (by Grötzsch's Theorem) in their construction. West [11] proposed a stronger version of Conjecture 1 in which only four slopes are allowed, thus using the fact that these graphs are 4-colorable.

Notice that two segments cross at most one point, whereas in the construction of Theorem 1, strings may cross twice. Let us define a 1 -string representation as a string representation in which any two strings cross at most once. Thus the following theorem is a step towards Conjecture 1.

\section{Theorem 2 Planar graphs have a 1-string representation.}

Note that if we would allow and consider tangent points, this theorem would directly follow from Koebe's theorem. Theorem 2 answers an open problem of de Fraysseix and Ossona de Mendez [5]. In the same article they noticed that Theorem 2 implies that any planar multigraph has a string representation such that the number of crossings between two strings equals the number of edges between the two corresponding vertices.

In the next section we provide some definitions and prove that it is sufficient to prove this theorem for triangulations. Section 3 is devoted to the study of string representations of 4-connected triangulations. In this section we use a decomposition technique of 4-connected triangulations that is inspired on Whitney's work [12] and that was recently used by the second author [7]. Then in Sect. 4 we finally prove Theorem 2 for all triangulations. 


\section{Preliminaries}

\subsection{Restriction to Triangulations}

Lemma 1 Every planar graph is an induced subgraph of some planar triangulation.

Proof Let $G$ be a planar graph embedded in the plane (i.e., a plane graph). The graph $h(G)$ is obtained from $G$ by adding in every face $f$ of $G$ a new vertex $v_{f}$ adjacent to every vertex incident to $f$ in $G$. Notice that $h(G)$ is also a plane graph and that $G$ is an induced subgraph of $h(G)$. Moreover, $h(G)$ is connected, $h(h(G))$ is 2-connected, and $h(h(h(G)))$ is a triangulation.

Note that we have to apply the $h$ operator several times: if a facial walk goes through the same vertex several times, since multiples edges are not allowed, we obtain a nontriangular face.

It is clear that a 1-string representation of a triangulation $T$ induces a 1-string representation for any of its induced subgraphs. It is thus sufficient to prove Theorem 2 for triangulations.

\subsection{String Representations}

In a plane graph $G$, the unbounded face of $G$ is called the outer-face and every other face of $G$ is an inner-face of $G$. An outer-vertex (resp. outer-edge) of $G$ is a vertex (resp. edge) of $G$ incident to the outer-face. The other vertices (resp. edges) of $G$ are inner-vertices (resp. inner-edges). The set of outer-vertices (resp. outer-edges, inner-vertices, and inner-edges) of $G$ is denoted by $V_{\mathrm{o}}(G)$ (resp. $E_{\mathrm{o}}(G), V_{\mathrm{i}}(G)$, and $\left.E_{\mathrm{i}}(G)\right)$. A near-triangulation is a plane graph in which all the inner-faces are triangles. An edge $u v$ is a chord of some near-triangulation $T$ if $u v$ is an inner-edge linking two outer-vertices. From now on, we use the following notation: the strings corresponding to vertices of a graph $G$ are denoted by bold letters, i.e., for any $v \in V(G)$, we denote its corresponding string by $\mathbf{v}$. We need that in a 1 -string representation of a plane graph $G$, each face of $G$ corresponds to some topological region of the string representation.

Definition 1 Let $G=(V, E)$ be a plane graph with a 1-string representation $\Sigma$. Given a face $a b c$ of $G$, consider a triplet $(a, b, c)$ of its incident vertices. An $(a, b, c)$ region abc is a region of the plane homeomorphic to a disk such that (see Fig. 1):

- For any vertex $v \neq a, b$, and $c$, we have $\mathbf{a b c} \cap \mathbf{v}=\emptyset$ (i.e., abc intersects only with $\mathbf{a}, \mathbf{b}, \mathbf{c})$.

- $\mathbf{a b c} \cap \mathbf{a} \cap \mathbf{b}=\emptyset, \mathbf{a b c} \cap \mathbf{b} \cap \mathbf{c}=\emptyset$, and $\mathbf{a b c} \cap \mathbf{c} \cap \mathbf{a}=\emptyset$ (i.e., $\mathbf{a}, \mathbf{b}, \mathbf{c}$ intersect outside abc).

- Both abc $\cap \mathbf{b}$ and $\mathbf{a b c} \cap \mathbf{c}$ are connected.

- The boundary of abc successively crosses (clockwise or anticlockwise) $\mathbf{a}, \mathbf{a}, \mathbf{b}, \mathbf{b}$, $\mathbf{c}, \mathbf{a}, \mathbf{c}$. 
Fig. 1 An $(a, b, c)$-region abc

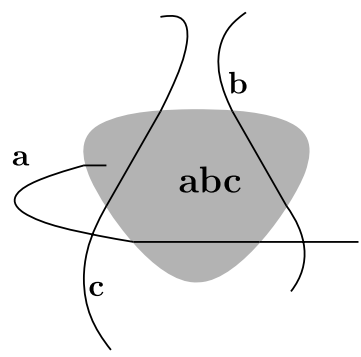

Note that according to this definition, abc $\cap \mathbf{a}$ has two components, and one end of $\mathbf{a}$ is in abc. Note that the order in the triplet $(a, b, c)$ matters: a region $\tau$ of the plane cannot be an $(a, b, c)$-region and a $(c, b, a)$-region for example. A region abc of the plane is an $\{a, b, c\}$-region if it is either an $(a, b, c)$-region, an $(a, c, b)$-region, a $(b, a, c)$-region, a $(b, c, a)$-region, a $(c, a, b)$-region, or a $(c, b, a)$-region. When the vertices $a, b$, and $c$ are not mentioned, we call such a region a face-region.

Definition 2 A strong 1-string representation (S-representation, for short) of a neartriangulation $T$ is a pair $(\Sigma, R)$ such that:

(1) $\Sigma$ is a 1-string representation of $T$.

(2) $R$ is a set of disjoint face-regions such that for every inner-face $a b c$ of $T, R$ contains an $\{a, b, c\}$-region.

A partial strong 1-string representation (PS-representation, for short) of a neartriangulation $T$ is a triplet $(\Sigma, R, F)$ in which $F \subseteq E(T)$ and such that $(\Sigma, R)$ is a strong 1-string representation of $T$ without the crossings corresponding to the edges of $F$.

In a PS-representation $(\Sigma, R, F)$ of $T$, note that $\Sigma$ is a 1-string representation of $T \backslash F$ and that each inner-face of $T$ has a corresponding face-region in $R$.

\subsection{Special Triangulations}

In a near-triangulation $T$, a separating 3-cycle $C$ is a cycle of length 3 such that some vertices of $T$ lie inside $C$, whereas other vertices lie outside. It is well known that a triangulation is 4-connected if and only if it contains no separating 3-cycle. In [12], Whitney considered a special family of near-triangulations, it is why we call them W-triangulations.

Definition 3 A $W$-triangulation is a 2-connected near-triangulation containing no separating 3-cycle.

In particular, any 4-connected triangulation is a W-triangulation. Note that since a W-triangulation has no cut vertex, its outer-edges induce a cycle. The following lemma gives a sufficient condition for a subgraph of a W-triangulation $T$ to be a W-triangulation. 
Fig. 2 3-boundary of $T$

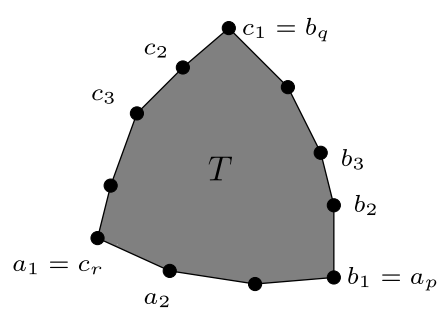

Lemma 2 Let $T$ be a $W$-triangulation and consider a cycle $C$ of $T$. The subgraph induced by the vertices lying on and inside $C$ is a $W$-triangulation.

Proof Consider the near-triangulation $T^{\prime}$ inside some cycle $C$ of $T$. By definition, $T$ has no separating 3-cycle, and consequently $T^{\prime}$ does not have any separating 3cycle. Since $T^{\prime}$ is clearly connected and has more than two vertices, we prove that it is 2-connected by showing that it does not contain any cut vertex.

Since the cycle $C$ delimits the outer-face of $T^{\prime}$, any vertex $v \in V\left(T^{\prime}\right)$ appears at most once on the outer face. Since the outerface appears at most once around $v$ and since all its other incident faces are triangles, $T^{\prime}$ contains a path linking all the neighbors of $v$. This implies that $T^{\prime} \backslash v$ is connected, and thus $T^{\prime}$ has no cut vertex.

Definition 4 A W-triangulation $T$ is 3-bounded if the outer-boundary of $T$ is the union of three paths $\left(a_{1}, \ldots, a_{p}\right),\left(b_{1}, \ldots, b_{q}\right)$, and $\left(c_{1}, \ldots, c_{r}\right)$ that satisfy the following conditions (see Fig. 2):

- $a_{1}=c_{r}, b_{1}=a_{p}$, and $c_{1}=b_{q}$.

- the paths are nontrivial, i.e., $p \geq 2, q \geq 2$, and $r \geq 2$.

- there exists no chord $a_{i} a_{j}, b_{i} b_{j}$, or $c_{i} c_{j}$.

Such a 3-boundary of $T$ will be denoted by $\left(a_{1}, \ldots, a_{p}\right)-\left(b_{1}, \ldots, b_{q}\right)-\left(c_{1}, \ldots, c_{r}\right)$.

In the following, we will use the order on the three paths and their directions, i.e., $\left(a_{1}, \ldots, a_{p}\right)-\left(b_{1}, \ldots, b_{q}\right)-\left(c_{1}, \ldots, c_{r}\right)$ will be different from $\left(b_{1}, \ldots, b_{q}\right)-$ $\left(c_{1}, \ldots, c_{r}\right)-\left(a_{1}, \ldots, a_{p}\right)$ and $\left(a_{p}, \ldots, a_{1}\right)-\left(c_{r}, \ldots, c_{1}\right)-\left(b_{q}, \ldots, b_{1}\right)$.

\section{Proof for 4-connected Triangulations}

The following property describes the shape of a PS-representation of a 3-bounded W-triangulation.

Property 1 Consider a 3-bounded $W$-triangulation $T$ with a 3-boundary $\left(a_{1}, \ldots\right.$, $\left.a_{p}\right)-\left(b_{1}, \ldots, b_{q}\right)-\left(c_{1}, \ldots, c_{r}\right)$. The $W$-triangulation $T$ has Property 1 if $T$ has a PSrepresentation $(\Sigma, R, F)$ contained inside a region $\tau$ of the plane homeomorphic to the disk that satisfies the following properties (see Fig. 3): 
Fig. 3 Property 1

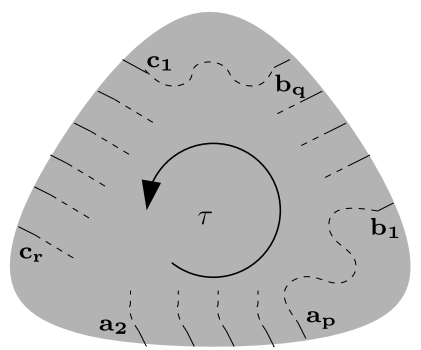

(a) $F=E_{\mathrm{o}}(T) \backslash\left\{a_{1} a_{2}\right\}$ (i.e., the missing crossings correspond to the outer edges, except $\left.a_{1} a_{2}\right)$.

(b) On the boundary of $\tau$, we successively have the ends of $\mathbf{a}_{2}, \mathbf{a}_{3}, \ldots, \mathbf{a}_{\mathbf{p}}, \mathbf{b}_{1}, \ldots$, $\mathbf{b}_{\mathbf{q}}, \mathbf{c}_{\mathbf{1}}, \ldots, \mathbf{c}_{\mathbf{r}}$.

If going clockwise (resp. anticlockwise) around the boundary of $\tau$, we cross the strings in the order described in (b), we say that the PS-representation is clockwise (resp. anticlockwise). Note that by an axial symmetry, one can obtain a clockwise PS-representation from an anticlockwise PS-representation, and vice versa. Observe that since $a_{p}=b_{1}, b_{q}=c_{1}$, and $c_{r}=a_{1}$, both ends of $\mathbf{b}_{1}$ and $\mathbf{c}_{1}$ lie on the boundary of $\tau$, but it is not the case for $\mathbf{a}_{1}$ or any other string (i.e., all the strings appearing on the boundary of $\tau$ have an end inside $\tau$ except $\mathbf{b}_{\mathbf{1}}$ and $\mathbf{c}_{\mathbf{1}}$ ).

Before proving that each 3-bounded $\mathrm{W}$-triangulation has Property 1 , we give some definitions and we present Property 2. Consider a 3-bounded W-triangulation $T \neq K_{3}$ whose boundary is $\left(a_{1}, \ldots, a_{p}\right)-\left(b_{1}, \ldots, b_{q}\right)-\left(c_{1}, \ldots, c_{r}\right)$ and such that $T$ does not contain any chord $a_{i} b_{j}$ or $a_{i} c_{j}$. Let $D \subseteq V_{\mathrm{i}}(T)$ be the set of inner-vertices of $T$ that are adjacent to some vertex $a_{\mathrm{i}}$ with $i>1$ (the black vertices on the left of Fig. 4). Since $T$ has at least 4 vertices, no separating 3 -cycle, and no chord $a_{i} a_{j}, a_{i} b_{j}$, or $a_{i} c_{j}$, it follows that $a_{1}$ and $a_{2}$ (resp. $b_{1}$ and $b_{2}$ ) have exactly one common neighbor in $V_{i}(T)$ that will be denoted $a$ (resp. $\left.d_{1}\right)$.

Since there is no chord $a_{i} a_{j}, a_{i} b_{j}$, or $a_{i} c_{j}$, for each vertex $a_{i}$ with $i \in[2, p-1]$, all the neighbors of $a_{i}$ (resp. $a_{p}$ ) except $a_{i-1}$ and $a_{i+1}$ (resp. $a_{p-1}$ and $b_{2}$ ) are in $D$. Since for each $i \in[2, p]$, there is a path linking the neighbors of $a_{i}$ in $D$ and since the vertices $a_{i}$ and $a_{i+1}$ have a common neighbor in $D$, the set $D$ induces a connected graph. Since $a$ is in $D$, the set $D \cup\left\{a_{1}\right\}$ also induces a connected graph.

Definition 5 The adjacent path of $T$ with respect to the 3-boundary $\left(a_{1}, \ldots, a_{p}\right)-$ $\left(b_{1}, \ldots, b_{q}\right)-\left(c_{1}, \ldots, c_{r}\right)$ is the shortest path linking $d_{1}$ and $a_{1}$ in $T\left[D \cup\left\{a_{1}\right\}\right]$ (the graph induced by $\left.D \cup\left\{a_{1}\right\}\right)$. This path will be denoted $\left(d_{1}, d_{2}, \ldots, d_{s}, a_{1}\right)$.

Observation 1 There exists neither an edge $d_{i} d_{j}$ with $2 \leq i+1<j \leq s$ nor an edge $a_{1} d_{i}$ with $1 \leq i<s$. Otherwise, $\left(d_{1}, d_{2}, \ldots d_{s}, a_{1}\right)$ would not be the shortest path between $d_{1}$ and $a_{1}$. 


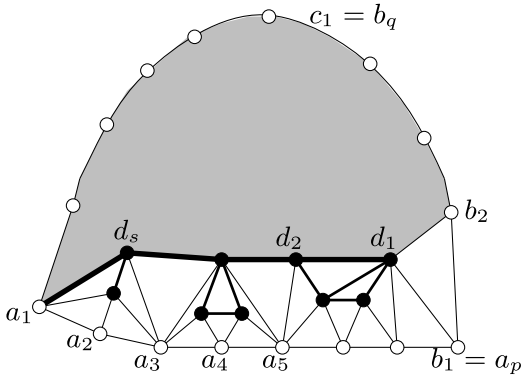

$T$

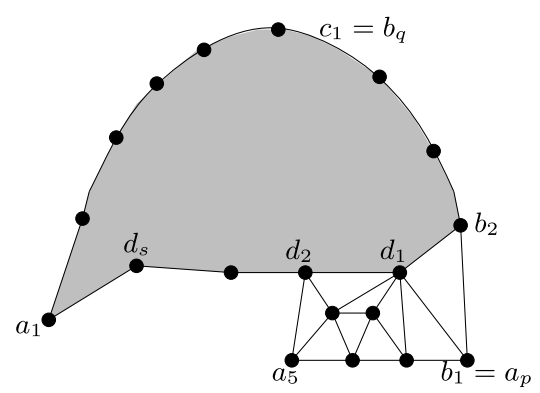

$T_{d_{2} a_{5}}$

Fig. 4 The adjacent path of $T$ and the graph $T_{d_{2} a_{5}}$

Definition 6 For each edge $d_{x} a_{y} \in E(T)$ with $x \in[1, s]$ and $y \in[2, p]$, the graph $T_{d_{x} a_{y}}$ is the graph lying inside the cycle $C=\left(a_{1}, d_{s}, \ldots, d_{x}, a_{y}, \ldots, a_{p}, b_{2}, \ldots\right.$, $b_{q}, c_{2}, \ldots, c_{r}$ ) (see Fig. 4).

Note that since $D \subseteq V_{\mathrm{i}}(T), C$ is a cycle, and by Lemma $2, T_{d_{x} a_{y}}$ is a $\mathrm{W}$ triangulation. The following property describes the shape of a PS-representation of $T_{d_{x} a_{y}}$.

Property 2 Consider a 3-bounded $W$-triangulation $T$ with a 3-boundary $\left(a_{1}, \ldots\right.$, $\left.a_{p}\right)-\left(b_{1}, \ldots, b_{q}\right)-\left(c_{1}, \ldots, c_{r}\right)$ that does not have any chord $a_{i} b_{j}$ or $a_{i} c_{j}$, and let $\left(d_{1}, d_{2}, \ldots, d_{s}, a_{1}\right)$ be its adjacent path. Consider an edge $d_{x} a_{y} \in E(T)$ with $y>1$.

The W-triangulation $T_{d_{x} a_{y}}$ has Property 2 if $T_{d_{x} a_{y}}$ has a PS-representation ( $\Sigma, R, F)$ satisfying the following properties (see Fig. 5):

(a) $F=E_{\mathrm{o}}(G) \backslash\left\{d_{x} a_{y}\right\}$.

(b) Every string $\mathbf{v} \in \Sigma \backslash\left\{\mathbf{d}_{\mathbf{x}}, \mathbf{a}_{\mathbf{y}}\right\}$ is contained in a region $\tau$ of the plane homeomorphic to the disk. Furthermore, $\mathbf{d}_{\mathbf{x}}$ and $\mathbf{a}_{\mathbf{y}}$ have their ends in $\tau$ (or on the boundary of $\tau$ ), but they cross each other outside $\tau$.

(c) Each face-region of $R$ is contained inside $\tau$.

(d) On the boundary of $\tau$, we successively have the ends of $\mathbf{a}_{\mathbf{y}}, \ldots, \mathbf{a}_{\mathbf{p}}, \mathbf{b}_{\mathbf{1}}, \ldots, \mathbf{b}_{\mathbf{q}}$, $\mathbf{c}_{1}, \ldots, \mathbf{c}_{\mathbf{r}}$,

$\mathbf{a}_{1}, \mathbf{d}_{\mathbf{s}}, \ldots, \mathbf{d}_{\mathbf{x}+\mathbf{1}}$, and then we successively have internal points of $\mathbf{d}_{\mathbf{x}}, \mathbf{a}_{\mathbf{y}}, \mathbf{d}_{\mathbf{x}}$, and $\mathbf{a}_{\mathbf{y}}$.

Here again, if going clockwise (resp. anticlockwise) around the boundary of $\tau$, we cross the strings in the order described in $(d)$, we say that the PS-representation is clockwise (resp. anticlockwise). In the proof of Theorem 2, we only use Property 1. However, in order to prove Property 1, we use Property 2. We prove these two properties by doing a "crossed" induction.

Proof of Properties 1 and 2

We prove, by induction on $m \geq 3$, that the following two statements hold: 
Fig. 5 Property 2

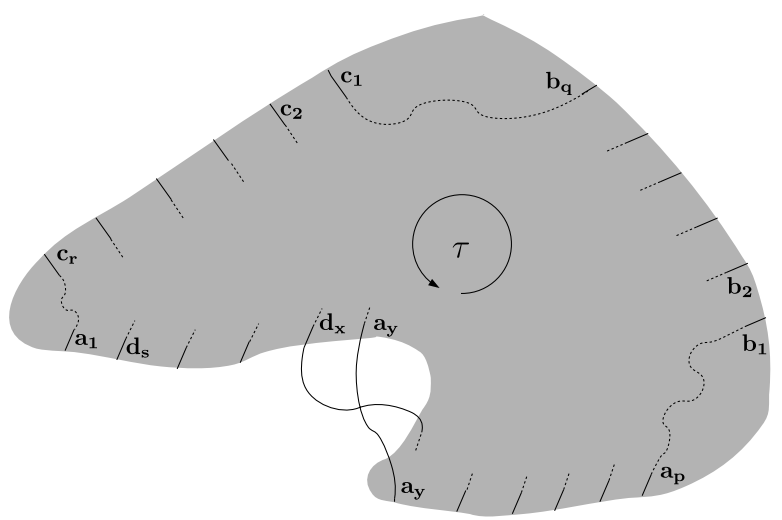

Fig. 6 Initial case for Property 1

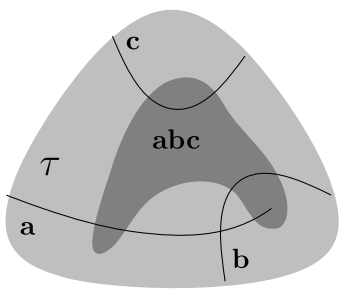

- Property 1 holds if $T$ has at most $m$ edges.

- Property 2 holds if $T_{d_{x} a_{y}}$ has at most $m$ edges.

The initial case, $m=3$, is easy to prove since there is only one $\mathrm{W}$-triangulation having at most 3 edges, $K_{3}$. For Property 1 , we have to consider all the possible 3-boundaries of $K_{3}$. All these 3-boundaries are equivalent, so let $V\left(K_{3}\right)=\{a, b, c\}$ and consider the 3-boundary $(a, b)-(b, c)-(c, a)$. In Fig. 6 there is a PS-representation $(\Sigma, R, F)$ of $K_{3}$ with $F=\{b c, a c\}$ that fulfills Property 1 . For Property 2, since a W-triangulation $T_{d_{x} a_{y}}$ has at least 4 vertices, $a_{1}, b_{1}, c_{1}$, and $d_{1}$, we have $T_{d_{x} a_{y}} \neq K_{3}$, and there is no W-triangulation $T_{d_{x} a_{y}}$ with at most 3 edges. So by vacuity, Property 2 holds for $T_{d_{x} a_{y}}$ with at most 3 edges.

The induction step applies to both Property 1 and Property 2 . This means that we prove Property 1 (resp. Property 2) for the W-triangulations $T$ (resp. $T_{d_{x} a_{y}}$ ) with $m$ edges using both Property 1 and Property 2 on W-triangulations with less than $m$ edges. We first prove the induction for Property 1.

Case 1: Proof of Property 1 for a $W$-triangulation $T$ with $m$ edges Let $\left(a_{1}, \ldots\right.$, $\left.a_{p}\right)-\left(b_{1}, \ldots, b_{q}\right)-\left(c_{1}, \ldots, c_{r}\right)$ be the 3-boundary of $T$ considered. We distinguish different cases according to the existence of a chord $a_{i} b_{j}$ or $a_{i} c_{j}$ in $T$. We successively consider the case where there is a chord $a_{1} b_{i}$ with $1<i<q$, the case where there is a chord $a_{i} b_{j}$ with $1<i<p$ and $1<j \leq q$, and the case where there is a chord $a_{i} c_{j}$ with $1<i \leq p$ and $1<j<r$. We then finish with the case where there is no chord $a_{i} b_{j}$ with $1 \leq i \leq p$ and $1 \leq j \leq q$ (by the definition of 3-boundary, $T$ has no chord $a_{1} b_{q}, a_{i} b_{1}$, or $\left.a_{p} b_{j}\right)$ and no chord $a_{i} c_{j}$ with $1 \leq i \leq p$ and $1 \leq j \leq r$ (by the definition of 3-boundary, $T$ has no chord $a_{p} c_{1}, a_{i} c_{r}$, or $a_{1} c_{j}$ ). 
Fig. 7 Case 1.1: Chord $a_{1} b_{i}$

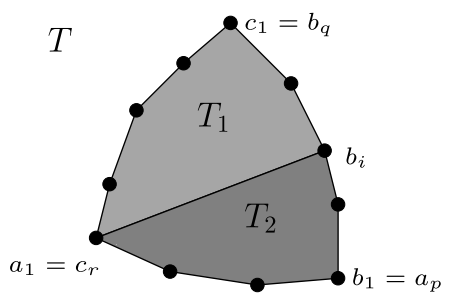

Fig. 8 Case 1.1: $(\Sigma, R, F)$

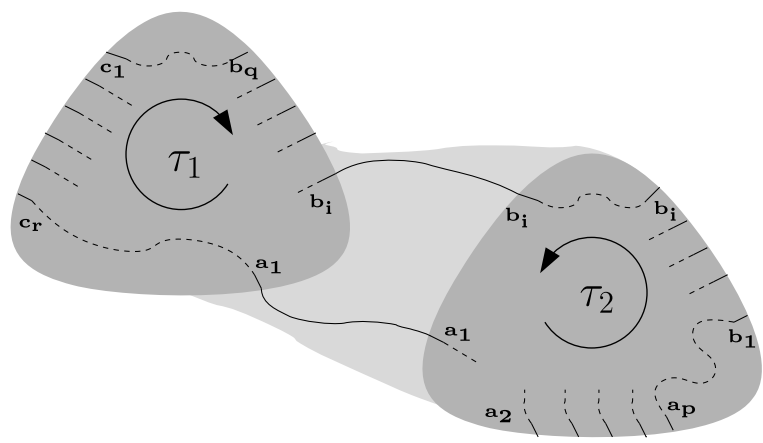

Case 1.1: There is a chord $a_{1} b_{\mathrm{i}}$ with $1<i<q$ (see Fig. 7) Let $T_{1}$ (resp. $T_{2}$ ) be the subgraph of $T$ that lies inside the cycle $\left(a_{1}, b_{i}, \ldots, b_{q}, c_{2}, \ldots, c_{r}\right)$ (resp. $\left.\left(a_{1}, a_{2}, \ldots, a_{p}, b_{2}, \ldots, b_{i}, a_{1}\right)\right)$. By Lemma $2, T_{1}$ and $T_{2}$ are W-triangulations. Since $T$ has no chord $a_{x} a_{y}, b_{x} b_{y}$, or $c_{x} c_{y},\left(b_{i}, a_{1}\right)-\left(c_{r}, \ldots, c_{1}\right)-\left(b_{q}, \ldots, b_{i}\right)$ (resp. $\left.\left(a_{1}, \ldots, a_{p}\right)-\left(b_{1}, \ldots, b_{i}\right)-\left(b_{i} a_{1}\right)\right)$ is a 3 -boundary of $T_{1}$ (resp. $\left.T_{2}\right)$. Furthermore, since $a_{1} a_{2} \notin E\left(T_{1}\right)$ and $c_{1} c_{2} \notin E\left(T_{2}\right), T_{1}$ and $T_{2}$ have less edges than $T$, and Property 1 holds for $T_{1}$ and $T_{2}$ with the mentioned 3-boundaries. Let $\left(\Sigma_{1}, R_{1}, F_{1}\right)$ (resp. $\left(\Sigma_{2}, R_{2}, F_{2}\right)$ ) be a clockwise (resp. anticlockwise) PS-representation contained in the region $\tau_{1}$ (resp. $\tau_{2}$ ) obtained for $T_{1}$ (resp. $T_{2}$ ) with $F_{1}=E_{\mathrm{o}}\left(T_{1}\right) \backslash\left\{a_{1} b_{i}\right\}$ (resp. $\left.F_{2}=E_{\mathrm{o}}\left(T_{2}\right) \backslash\left\{a_{1} a_{2}\right\}\right)$. In Fig. 8 we show how to associate these two representations to obtain $(\Sigma, R, F)$, an anticlockwise PS-representation of $T$ contained in $\tau$. Note that the two strings $\mathbf{a}_{\mathbf{1}}$ (resp. $\mathbf{b}_{\mathbf{i}}$ ) from $\Sigma_{1}$ and $\Sigma_{2}$ have been linked.

We easily verify that $(\Sigma, R, F)$ satisfies Property 1 :

- $\Sigma$ is a string representation of $T \backslash F$ with $F=E_{\mathrm{o}}(T) \backslash\left\{a_{1} a_{2}\right\}$. Indeed, since $V\left(T_{1}\right) \cup V\left(T_{2}\right)=V(T)$ and $V\left(T_{1}\right) \cap V\left(T_{2}\right)=\left\{a_{1}, b_{i}\right\}$, every vertex $v \in V(T)$ has exactly one string in $\Sigma$. Furthermore, since $\left(E\left(T_{1}\right) \backslash F_{1}\right) \cup\left(E\left(T_{2}\right) \backslash F_{2}\right)=$ $E(T) \backslash F, \Sigma$ is a string representation of $T \backslash F$.

- $\Sigma$ is a 1-string representation. The only edge that belongs to both $T_{1}$ and $T_{2}$ is $a_{1} b_{i}$. Since $\mathbf{a}_{1}$ and $\mathbf{b}_{\mathbf{i}}$ cross each other in $\Sigma_{1}\left(a_{1} b_{i} \notin F_{1}\right)$ but not in $\Sigma_{2}\left(a_{1} b_{i} \in F_{2}\right)$, $\mathbf{a}_{\mathbf{1}}$ and $\mathbf{b}_{\mathbf{i}}$ cross exactly once in $\Sigma$.

- $(\Sigma, R)$ is "strong": Each inner-face of $T$ is an inner-face in $T_{1}$ or $T_{2}$, and the regions $\tau_{1}$ and $\tau_{2}$ are disjoint (so the face-regions in $\tau_{1}$ are disjoint from the faceregions in $\tau_{2}$ ).

Finally we see in Fig. 8 that point $(b)$ of Property 1 is satisfied. 
Fig. 9 Case 1.2: Chord $a_{i} b_{j}$

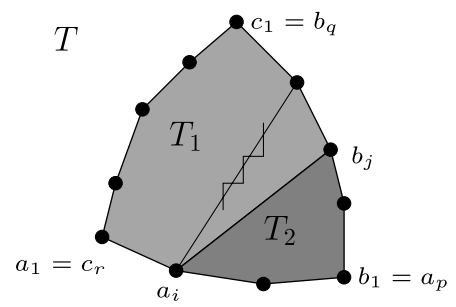

Fig. 10 Case 1.2: $(\Sigma, R, F)$

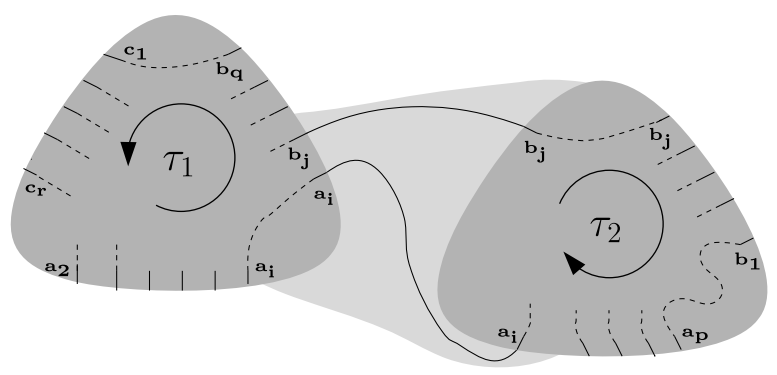

Case 1.2: There is a chord $a_{i} b_{j}$ with $1<i<p$ and $1<j \leq q$ (see Fig. 9) If there are several chords $a_{i} b_{j}$, we consider one that maximizes $j$, i.e., there is no chord $a_{i} b_{k}$ with $j<k \leq q$. Let $T_{1}$ (resp. $T_{2}$ ) be the subgraph of $T$ that lies inside the cycle $\left(a_{1}, a_{2}, \ldots, a_{i}, b_{j}, \ldots, b_{q}, c_{2}, \ldots, c_{r}\right)\left(\operatorname{resp} .\left(a_{i}, \ldots, a_{p}, b_{2}, \ldots, b_{j}, a_{i}\right)\right)$. By Lemma 2, $T_{1}$ and $T_{2}$ are W-triangulations. Since $T$ has no chord $a_{x} a_{y}, b_{x} b_{y}$, $c_{x} c_{y}$, or $a_{i} b_{k}$ with $k>j,\left(a_{1}, \ldots, a_{i}\right)-\left(a_{i}, b_{j}, \ldots, b_{q}\right)-\left(c_{1}, \ldots, c_{r}\right)\left(\operatorname{resp} .\left(a_{i}, b_{j}\right)-\right.$ $\left.\left(b_{j}, \ldots, b_{1}\right)-\left(a_{p}, \ldots, a_{i}\right)\right)$ is a 3-boundary of $T_{1}$ (resp. $\left.T_{2}\right)$. Furthermore, since $b_{1} b_{2} \notin$ $E\left(T_{1}\right)$ and $a_{1} a_{2} \notin E\left(T_{2}\right), T_{1}$ and $T_{2}$ have less edges than $T$, and Property 1 holds for $T_{1}$ and $T_{2}$ with the mentioned 3-boundaries. Let $\left(\Sigma_{1}, R_{1}, F_{1}\right)$ (resp. $\left.\left(\Sigma_{2}, R_{2}, F_{2}\right)\right)$ be an anticlockwise (resp. clockwise) PS-representation contained in the region $\tau_{1}$ (resp. $\left.\tau_{2}\right)$ obtained for $T_{1}\left(\operatorname{resp} . T_{2}\right)$ with $F_{1}=E_{\mathrm{o}}\left(T_{1}\right) \backslash\left\{a_{1} a_{2}\right\}$ (resp. $\left.F_{2}=E_{\mathrm{o}}\left(T_{2}\right) \backslash\left\{a_{i} b_{j}\right\}\right)$. In Fig. 10 we show how to associate these two representations to obtain $(\Sigma, R, F)$, an anticlockwise PS-representation of $T$ contained in $\tau$. Note that in this construction the two strings $\mathbf{a}_{\mathbf{i}}$ (resp. $\mathbf{b}_{\mathbf{j}}$ ) from $\Sigma_{1}$ and $\Sigma_{2}$ have been linked.

As in Case 1.1, we easily verify that $(\Sigma, R, F)$ satisfies Property 1 .

Case 1.3: There is a chord $a_{i} c_{j}$ with $1<i \leq p$ and $1<j<r$ (see Fig. 11) If there are several chords $a_{i} c_{j}$, we consider one which maximizes $i$, i.e., there is no chord $a_{k} c_{j}$ with $i<k \leq p$. Let $T_{1}$ (resp. $T_{2}$ ) be the subgraph of $T$ that lies inside the cycle $\left(a_{1}, a_{2}, \ldots, a_{i}, c_{j}, \ldots, c_{r}\right)$ (resp. $\left.\left(c_{j}, a_{i}, \ldots, a_{p}, b_{2}, \ldots, b_{q}, c_{2}, \ldots, c_{j}\right)\right)$. By Lemma 2, $T_{1}$ and $T_{2}$ are W-triangulations. Since $T$ has no chord $a_{x} a_{y}, b_{x} b_{y}$, $c_{x} c_{y}$, or $a_{k} c_{j}$ with $k>i,\left(a_{1}, \ldots, a_{i}\right)-\left(a_{i}, c_{j}\right)-\left(c_{j}, \ldots, c_{r}\right)\left(\operatorname{resp} .\left(c_{j}, a_{i}, \ldots, a_{p}\right)-\right.$ $\left.\left(b_{1}, \ldots, b_{q}\right)-\left(c_{1}, \ldots, c_{j}\right)\right)$ is a 3-boundary of $T_{1}$ (resp. $\left.T_{2}\right)$. Furthermore, since $b_{1} b_{2} \notin$ $E\left(T_{1}\right)$ and $a_{1} a_{2} \notin E\left(T_{2}\right), T_{1}$ and $T_{2}$ have less edges than $T$, and Property 1 holds for $T_{1}$ and $T_{2}$ with the mentioned 3-boundaries. Let $\left(\Sigma_{1}, R_{1}, F_{1}\right)$ (resp. $\left.\left(\Sigma_{2}, R_{2}, F_{2}\right)\right)$ be an anticlockwise PS-representation contained in the region $\tau_{1}$ (resp. $\tau_{2}$ ) obtained for $T_{1}$ (resp. $T_{2}$ ) with $F_{1}=E_{\mathrm{o}}\left(T_{1}\right) \backslash\left\{a_{1} a_{2}\right\}$ (resp. $\left.F_{2}=E_{\mathrm{o}}\left(T_{2}\right) \backslash\left\{c_{j} a_{i}\right\}\right)$. In Fig. 12 we 
Fig. 11 Case 1.3: Chord $a_{i} c_{j}$

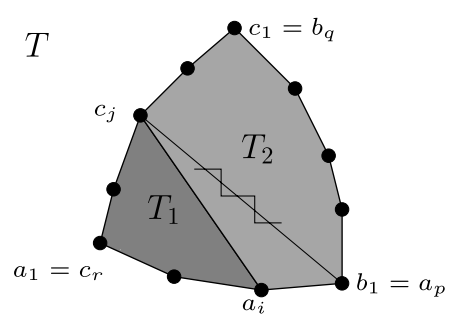

Fig. 12 Case 1.3: $(\Sigma, R, F)$

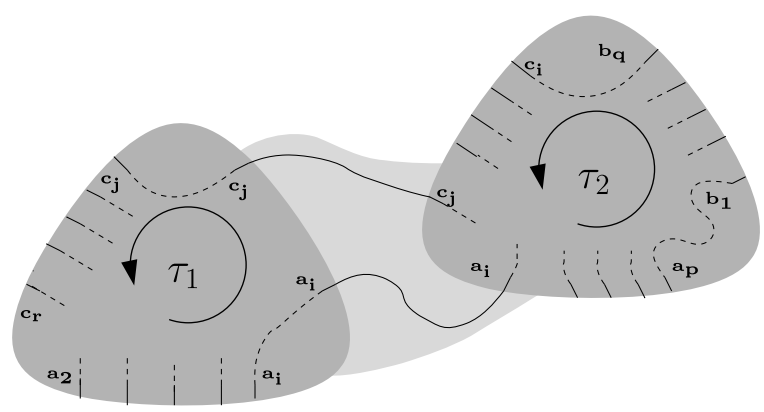

show how to associate these two representations to obtain $(\Sigma, R, F)$, an anticlockwise PS-representation of $T$ contained in $\tau$. Note that in this construction the two strings $\mathbf{a}_{\mathbf{i}}$ (resp. $\mathbf{c}_{\mathbf{j}}$ ) from $\Sigma_{1}$ and $\Sigma_{2}$ have been linked.

As in Case 1.1 , we easily verify that $(\Sigma, R, F)$ satisfies Property 1 .

Case 1.4: There is no chord $a_{i} b_{j}$ with $1 \leq i \leq p$ and $1 \leq j \leq q$, and no chord $a_{i} c_{j}$ with $1 \leq i \leq p$ and $1 \leq j \leq r$ (see Fig. 13) In this case we consider the adjacent path $\left(d_{1}, \ldots, d_{s}, a_{1}\right)$ (see Fig. 4$)$ of $T$ with respect to its 3-boundary, $\left(a_{1}, \ldots, a_{p}\right)-$ $\left(b_{1}, \ldots, b_{q}\right)-\left(c_{1}, \ldots, c_{r}\right)$. Consider the edge $d_{s} a_{y}$ with $1<y \leq p$ and which minimizes $y$. This edge exists since, by the definition of the adjacent path, $d_{s}$ is adjacent to some vertex $a_{y}$ with $y>1$. The $\mathrm{W}$-triangulation $T_{d_{s} a_{y}}$ having less edges than $T\left(a_{1} a_{2} \notin E\left(T_{d_{s} a_{y}}\right)\right)$, Property 2 holds for $T_{d_{s} a_{y}}$. Let $\left(\Sigma^{\prime}, R^{\prime}, F^{\prime}\right)$ be an anticlockwise PS-representation almost contained in the region $\tau^{\prime}$ obtained for $T_{d_{s} a_{y}}$, with $F^{\prime}=E_{\mathrm{o}}\left(T_{d_{s} a_{y}}\right) \backslash\left\{d_{s} a_{y}\right\}$.

Now we distinguish two cases according to the position of $a_{y}$ : either $y=2$ (Case 1.4.1), or $y>2$ (Case 1.4.2).

Case 1.4.1: $y=2$ In Fig. 14, starting from $\left(\Sigma^{\prime}, R^{\prime}, F^{\prime}\right)$, we show how to extend the string $\mathbf{a}_{1} \in \Sigma^{\prime}$ (in order to cross $\mathbf{d}_{\mathbf{s}}$ and $\left.\mathbf{a}_{2}\right)$ and how to draw the $\left(a_{1}, a_{2}, d_{s}\right)$-region $\mathbf{a}_{1} \mathbf{a}_{2} \mathbf{d}_{\mathbf{s}}$ to obtain $(\Sigma, R, F)$, an anticlockwise PS-representation of $T$ contained in a region $\tau$.

One can verify on Fig. 14 that $(\Sigma, R, F)$ satisfies Property 1.

Case 1.4.2: $y>2$ Let us denote by $e_{1}, e_{2}, \ldots, e_{t}$ the neighbors of $d_{s}$ strictly inside the cycle $\left(d_{s}, a_{1}, a_{2}, \ldots, a_{y}, d_{s}\right)$, going "from right to left" (see Fig. 13). By minimality of $y$ we have $e_{i} \neq a_{j}$ for all $1 \leq i \leq t$ and $1 \leq j \leq y$. 

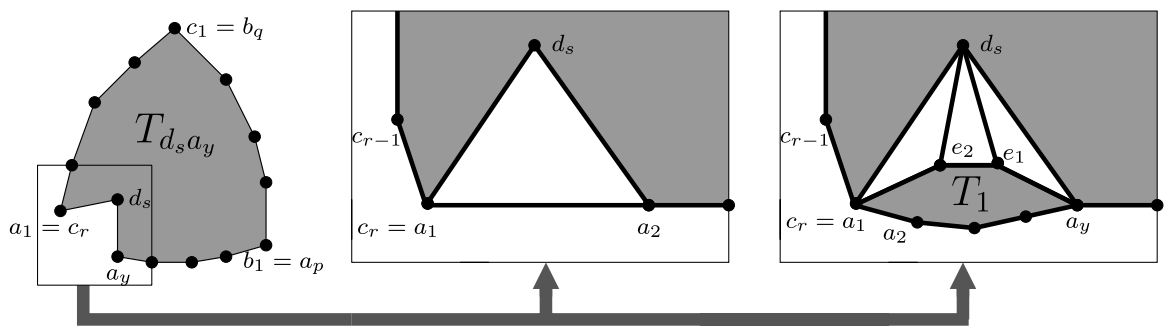

Fig. 13 Case 1.4: No chord $a_{i} b_{j}$ or $a_{i} c_{j}$

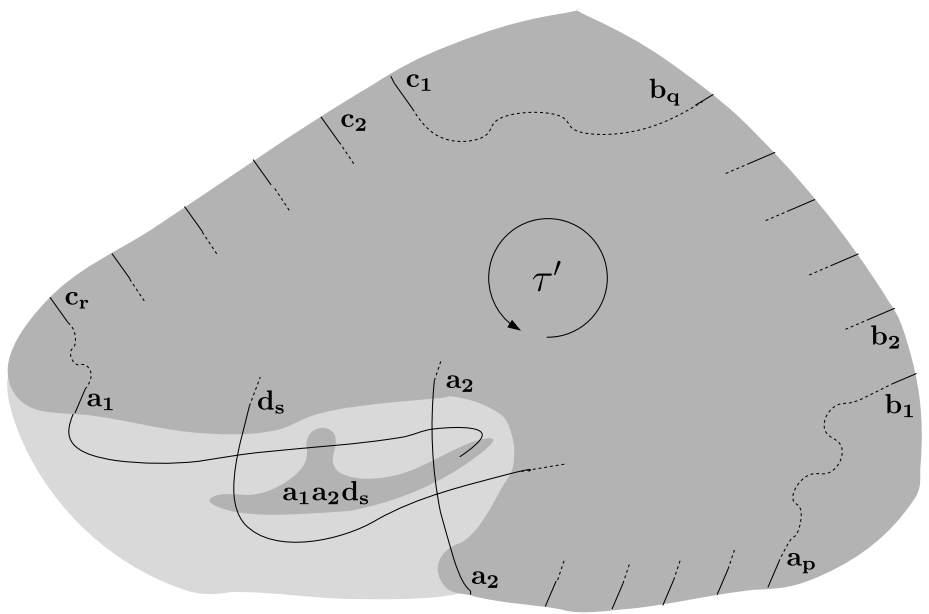

Fig. 14 Case 1.4.1

Let $T_{1}$ be the subgraph of $T$ that lies inside the cycle $\left(a_{1}, \ldots, a_{y}, e_{1}, \ldots, e_{t}, a_{1}\right)$. By Lemma 2, $T_{1}$ is a W-triangulation. Since the $\mathrm{W}$-triangulation $T$ has no separating 3-cycle $\left(d_{s}, a_{1}, e_{i}\right),\left(d_{s}, a_{y}, e_{i}\right)$, or $\left(d_{s}, e_{i}, e_{j}\right)$, there exists no chord $a_{1} e_{i}, a_{y} e_{i}$, or $e_{i} e_{j}$ in $T_{1}$. So $\left(a_{2}, a_{1}\right)-\left(a_{1}, e_{t}, \ldots, e_{1}, a_{y}\right)-\left(a_{y}, \ldots, a_{2}\right)$ is a 3 -boundary of $T_{1}$. Finally, since $T_{1}$ has less edges than $T\left(a_{1} d_{s} \notin E\left(T_{1}\right)\right)$, Property 1 holds for $T_{1}$ with respect to the mentioned 3-boundary. Let $\left(\Sigma_{1}, R_{1}, F_{1}\right)$ be a clockwise PS-representation contained in the region $\tau_{1}$ obtained for $T_{1}$ with $F_{1}=E_{0}\left(T_{1}\right) \backslash\left\{a_{2} a_{1}\right\}$.

In Fig. 15, starting from $\left(\Sigma^{\prime}, R^{\prime}, F^{\prime}\right)$ and $\left(\Sigma_{1}, R_{1}, F_{1}\right)$, we show how to join the strings $\mathbf{a}_{1}$ (resp. $\mathbf{a}_{\mathbf{y}}$ ) of $\Sigma^{\prime}$ and $\Sigma_{1}$, how to extend the strings $\mathbf{e}_{\mathbf{i}}$ for $1 \leq i \leq t$, and how to draw the face-regions $\mathbf{a}_{\mathbf{y}} \mathbf{e}_{\mathbf{1}} \mathbf{d}_{\mathbf{s}}, \mathbf{e}_{\mathbf{t}} \mathbf{a}_{\mathbf{1}} \mathbf{d}_{\mathbf{s}}$, and $\mathbf{e}_{\mathbf{i}} \mathbf{e}_{\mathbf{i}-\mathbf{1}} \mathbf{d}_{\mathbf{s}}$ for $2 \leq i \leq t$, in order to obtain $(\Sigma, R, F)$, an anticlockwise PS-representation of $T$ contained in a region $\tau$.

We verify that $(\Sigma, R, F)$ satisfies Property 1 :

- $\Sigma$ is a string representation of $T \backslash F$ with $F=E_{\mathrm{o}}(T) \backslash\left\{a_{1} a_{2}\right\}$. Indeed, since $V\left(T_{d_{s} a_{y}}\right) \cup V\left(T_{1}\right)=V(T)$ and $V\left(T_{d_{s} a_{y}}\right) \cap V\left(T_{1}\right)=\left\{a_{1}, a_{y}\right\}$, every vertex $v \in$ $V(T)$ has exactly one string in $\Sigma$. Furthermore, since $E(T) \backslash F=\left(E\left(T_{d_{s} a_{y}}\right) \backslash\right.$ $\left.F^{\prime}\right) \cup\left(E\left(T_{1}\right) \backslash F_{1}\right) \cup\left\{a_{y} e_{1}, e_{t} a_{1}, d_{s} a_{1}\right\} \cup\left\{e_{i} e_{i-1} \mid i \in[2, t]\right\} \cup\left\{d_{s} e_{\mathrm{i}} \mid i \in[1, t]\right\}, \Sigma$ is a string representation of $T \backslash F$. 


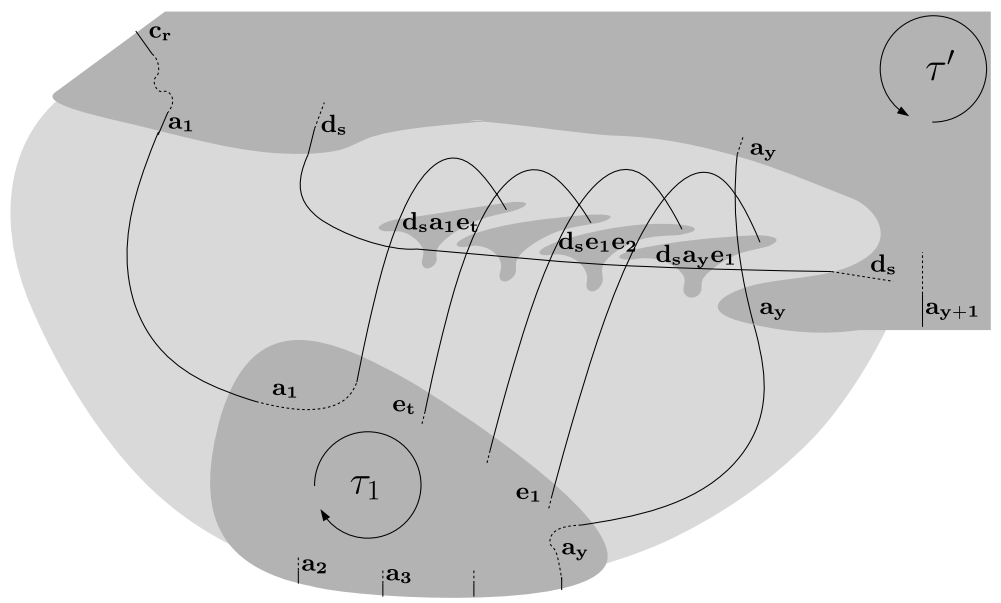

Fig. 15 Case 1.4.2

- $\Sigma$ is a 1-string representation. Indeed $T_{d_{s} a_{y}}$ and $T_{1}$ do not have common edges, and the new crossings added correspond to edges missing in both $E\left(T_{d_{s} a_{y}}\right) \backslash F^{\prime}$ and $E\left(T_{1}\right) \backslash F_{1}$.

- $(\Sigma, R)$ is "strong": The only inner-faces of $T$ not in $T_{d_{s} a_{y}}$ nor in $T_{1}$ are the faces $d_{s} a_{y} e_{1}, d_{s} a_{1} e_{t}$, and $d_{s} e_{i} e_{i+1}$ with $1 \leq i<t$. These faces correspond to the new face-regions.

Finally we see in Fig. 15 that point $(b)$ of Property 1 is satisfied.

So Property 1 holds for any $\mathrm{W}$-triangulation $T$ with $m$ edges, and this concludes the proof of Case 1 .

Case 2: Proof of Property 2 for a W-triangulation $T_{d_{x} a_{y}}$ with $m$ edges Recall that the W-triangulation $T_{d_{x} a_{y}}$ is a subgraph of a W-triangulation $T$ with 3-boundary $\left(a_{1}, \ldots, a_{p}\right)-\left(b_{1}, \ldots, b_{q}\right)-\left(c_{1}, \ldots, c_{r}\right)$. Moreover, $T$ has no chord $a_{i} b_{j}$ or $a_{i} c_{j}$, and its adjacent path is $\left(d_{1}, \ldots, d_{s}, a_{1}\right)$, with $s \geq 1$. We distinguish the case where $d_{x} a_{y}=d_{1} a_{p}$ and the case where $d_{x} a_{y} \neq d_{1} a_{p}$.

Case 2.1: $d_{x} a_{y}=d_{1} a_{p}$ (see Fig. 16) Let $T_{1}$ be the subgraph of $T_{d_{1} a_{p}}$ that lies inside the cycle $\left(a_{1}, d_{s}, \ldots, d_{1}, b_{2}, \ldots, b_{q}, c_{2}, \ldots, c_{r}\right)$. By Lemma $2, T_{1}$ is a Wtriangulation. This $\mathrm{W}$-triangulation has no chord $b_{i} b_{j}, c_{i} c_{j}, d_{i} d_{j}$, or $a_{1} d_{j}$. We consider two cases according to the existence of an edge $d_{1} b_{i}$ with $2<i \leq q$.

- If $T_{1}$ has no chord $d_{1} b_{i}$, then $\left(d_{1}, b_{2}, \ldots, b_{q}\right)-\left(c_{1}, \ldots, c_{r}\right)-\left(a_{1}, d_{s}, \ldots, d_{1}\right)$ is a 3 boundary of $T_{1}$.

- If $T_{1}$ has a chord $d_{1} b_{i}$ with $2<i \leq q$, note that $q>2$ and that there cannot be a chord $b_{2} a_{1}$ or $b_{2} d_{j}$ with $1<j \leq s$ (this would violate the planarity of $T_{d_{x} a_{y}}$, see Fig. 16). So in this case, $\left(b_{2}, d_{1}, \ldots, d_{s}, a_{1}\right)-\left(c_{r}, \ldots, c_{1}\right)-\left(b_{q}, \ldots, b_{2}\right)$ is a 3 boundary of $T_{1}$.

Finally, since $T_{1}$ is a W-triangulation with less edges than $T_{d_{1} a_{p}}\left(b_{1} b_{2} \notin E\left(T_{1}\right)\right)$, Property 1 holds for $T_{1}$ with respect to at least one of the two mentioned 3-boundaries. 
Fig. 16 Case 2.1:

$T_{d_{x} a_{y}}=T_{d_{1} a_{p}}$

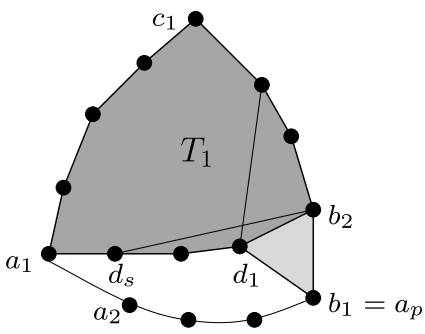

Fig. 17 Case 2.1: $(\Sigma, R, F)$

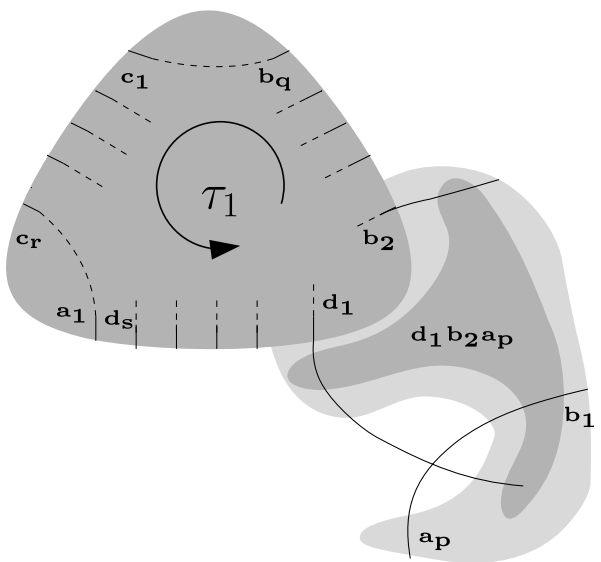

Whichever 3-boundary we consider, we obtain a PS-representation $\left(\Sigma_{1}, R_{1}, F_{1}\right)$ of $T_{1}$ contained in a region $\tau_{1}$, with the same following characteristics:

- $F_{1}=E_{\mathrm{o}}(T) \backslash\left\{d_{1} b_{2}\right\}$,

- in the boundary of $\tau_{1}$, we successively meet the ends of $\mathbf{d}_{\mathbf{1}}, \ldots, \mathbf{d}_{\mathbf{s}}, \mathbf{a}_{\mathbf{1}}, \mathbf{c}_{\mathbf{r}}, \ldots, \mathbf{c}_{\mathbf{1}}$, $\mathbf{b}_{\mathbf{q}}, \ldots, \mathbf{b}_{\mathbf{2}}$ (clockwise or anticlockwise).

In Fig. 17 we modify ( $\Sigma_{1}, R_{1}, F_{1}$ ), by extending the strings $\mathbf{d}_{\mathbf{1}}$ and $\mathbf{b}_{\mathbf{2}}$ and by adding a new string $\mathbf{a}_{\mathbf{p}}$ and a new face-region $\mathbf{d}_{\mathbf{1}} \mathbf{b}_{\mathbf{2}} \mathbf{a}_{\mathbf{p}}$. This leads to $(\Sigma, R, F)$, a PS-representation of $T_{d_{1} a_{p}}$ contained in a region $\tau$.

We verify that $(\Sigma, R, F)$ satisfies Property 2 :

- $\Sigma$ is a 1-string representation of $T_{d_{1} a_{p}} \backslash F$ : Indeed, $E\left(T_{d_{1} a_{p}}\right) \backslash F$ is the disjoint union of $E\left(T_{1}\right) \backslash F_{1}$ and $\left\{a_{p} d_{1}\right\}$.

- $(\Sigma, R)$ is "strong": The only inner-face of $T_{d_{1} a_{p}}$ that is not an inner-face of $T_{1}$ is $d_{1} a_{p} b_{2}$, which corresponds to the new face-region $\mathbf{d}_{\mathbf{1}} \mathbf{a}_{\mathbf{p}} \mathbf{b}_{\mathbf{2}}$.

Finally we see in Fig. 17 that the other points of Property 2 are satisfied.

Case 2.2: $T_{d_{x} a_{y}} \neq T_{d_{1} a_{p}}$ In this case we consider an edge $d_{z} a_{w} \in E\left(T_{d_{x} a_{y}}\right)$ such that $d_{z} a_{w} \neq d_{x} a_{y}$. Among all the possible edges $d_{z} a_{w}$, we choose the one that first maximizes $z$ and then minimizes $w$. Such an edge necessarily exists, and actually one can see that $d_{z}=d_{x}$ or $d_{z}=d_{x-1}$. Indeed, if $d_{x}=d_{1}$, there is at least one edge $d_{1} a_{w}$ with $w>y$, the edge $d_{1} a_{p}$. If $x>1$, it is clear by the definition of the adjacent path that the vertex $d_{x-1}$ is adjacent to at least one vertex $a_{w}$ with $w \geq y$. 


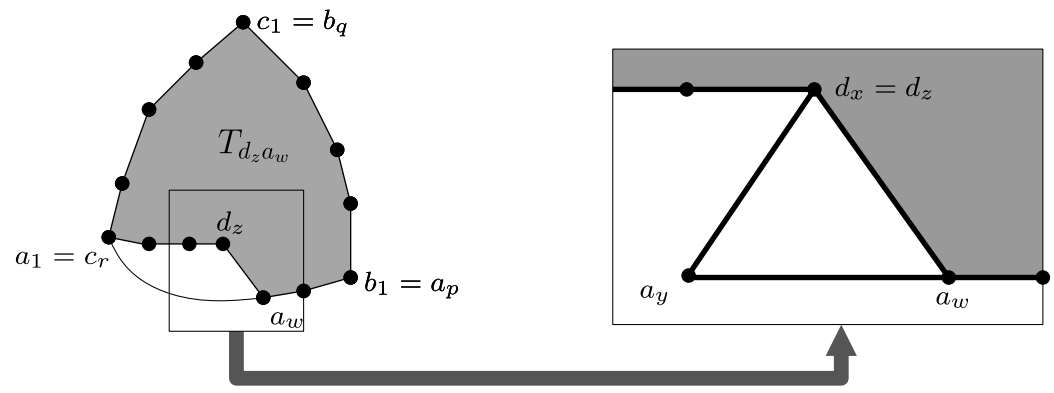

Fig. 18 Case 2.2.1: $z=x$ and $w=y+1$

Fig. 19 Case 2.2.1: $(\Sigma, R, F)$

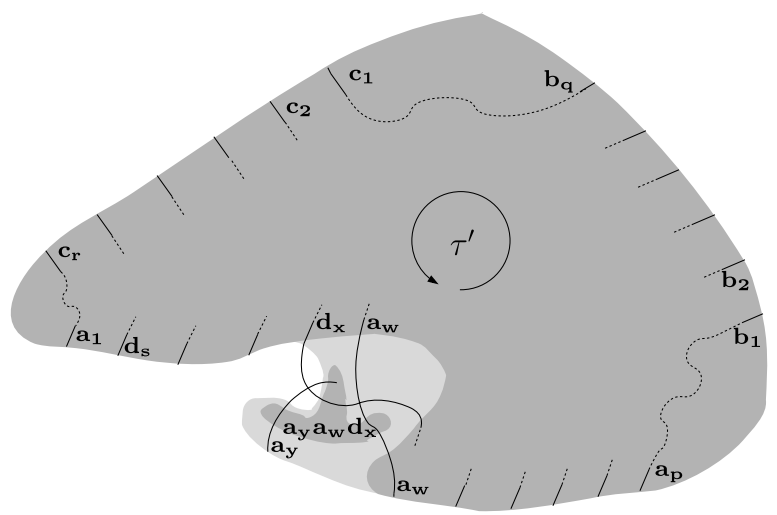

By Lemma 2, $T_{d_{z} a_{w}}$ is a W-triangulation. Since $d_{x} a_{y} \notin E\left(T_{d_{z} a_{w}}\right)$, the Wtriangulation $T_{d_{z} a_{w}}$ has less edges than $T_{d_{x} a_{y}}$, and so Property 2 holds for $T_{d_{z} a_{w}}$. Let $\left(\Sigma^{\prime}, R^{\prime}, F^{\prime}\right)$ be an anticlockwise PS-representation almost contained in the region $\tau^{\prime}$ obtained for $T_{d_{z} a_{w}}$ with $F^{\prime}=E_{0}\left(T_{d_{z} a_{w}}\right) \backslash\left\{d_{z} a_{w}\right\}$.

We distinguish four cases according to the edge $d_{z} a_{w}$. When $z=x$, we consider the case where $w=y+1$ and the case where $w>y+1$. When $z=x-1$, we consider the case where $w=y$ and the case where $w>y$.

Case 2.2.1: $T_{d_{x} a_{y}} \neq T_{d_{1} a_{p}}, z=x$, and $w=y+1$ (see Fig. 18) In Fig. 19 we modify $\left(\Sigma^{\prime}, R^{\prime}, F^{\prime}\right)$ by adding a new string $\mathbf{a}_{\mathbf{y}}$ and a new face-region $\mathbf{a}_{\mathbf{y}} \mathbf{a}_{\mathbf{w}} \mathbf{d}_{\mathbf{x}}$. This leads to $(\Sigma, R, F)$, an anticlockwise PS-representation of $T_{d_{x} a_{y}}$ almost contained in a region $\tau$.

We verify that $(\Sigma, R, F)$ satisfies Property 2 :

- $\Sigma$ is a 1-string representation of $T_{d_{x} a_{y}} \backslash F$ : Indeed, $E\left(T_{d_{x} a_{y}}\right) \backslash F$ is the disjoint union of $E\left(T_{d_{z} a_{w}}\right) \backslash F^{\prime}$ and $\left\{d_{x} a_{y}\right\}$.

- $(\Sigma, R)$ is "strong": The only inner-face of $T_{d_{x} a_{y}}$ that is not an inner-face of $T_{d_{z} a_{w}}$ is $d_{x} a_{y} a_{w}$, which corresponds to the new face-region $\mathbf{d}_{\mathbf{x}} \mathbf{a}_{\mathbf{y}} \mathbf{a}_{\mathbf{w}}$.

Finally we see in Fig. 19 that the other points of Property 2 are satisfied. 
Fig. 20 Case 2.2.2:

$T_{d_{x} a_{y}} \neq T_{d_{1} a_{p}}, z=x-1$, and $w=y$
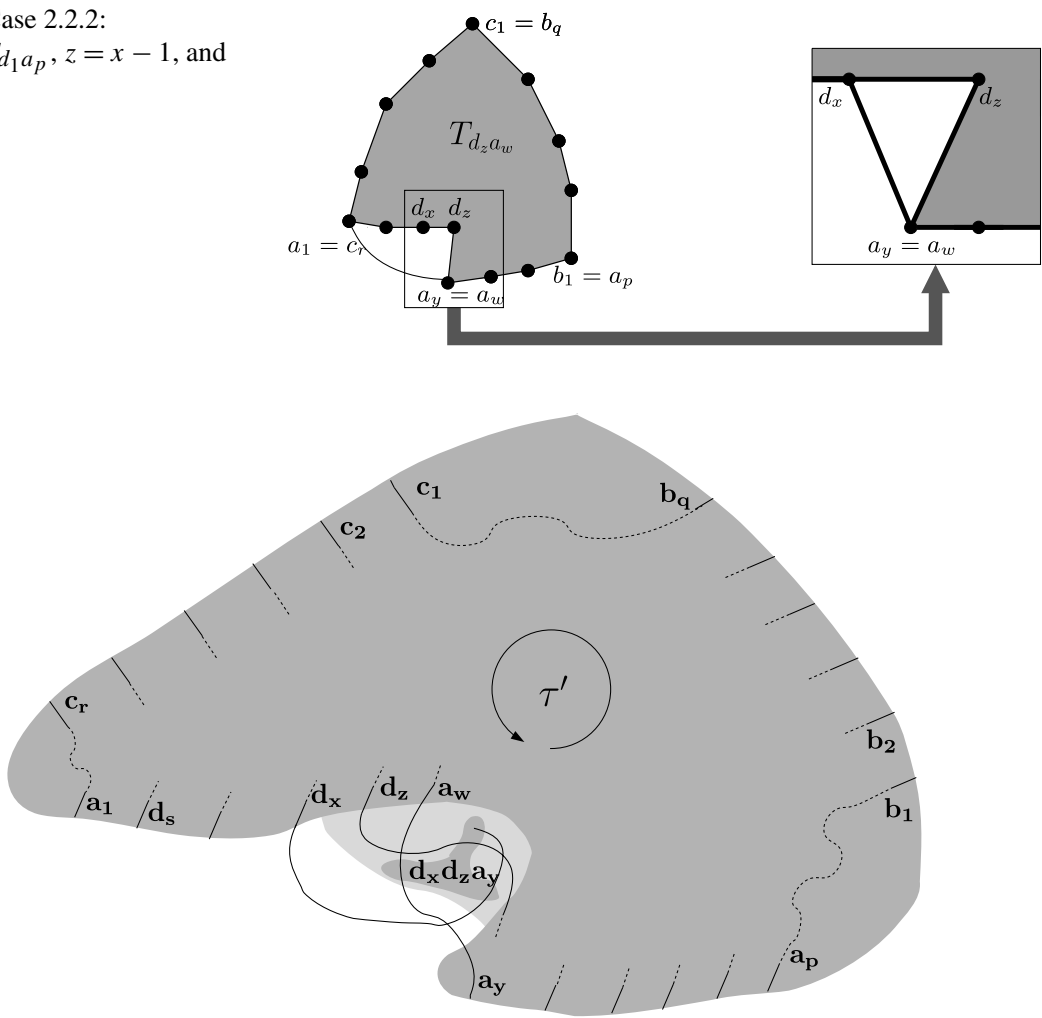

Fig. 21 Case 2.2.2: $(\Sigma, R, F)$

Case 2.2.2: $z=x-1$ and $w=y$ (see Fig. 20) In Fig. 21, we modify $\left(\Sigma^{\prime}, R^{\prime}, F^{\prime}\right)$ by extending the string $\mathbf{d}_{\mathbf{x}}$ and by adding a new face-region $\mathbf{d}_{\mathbf{x}} \mathbf{d}_{\mathbf{z}} \mathbf{a}_{\mathbf{y}}$. This leads to $(\Sigma, R, F)$, an anticlockwise PS-representation of $T_{d_{x} a_{y}}$ almost contained in a region $\tau$.

We verify that $(\Sigma, R, F)$ satisfies Property 2 :

- $\Sigma$ is a 1-string representation of $T_{d_{x} a_{y}} \backslash F$ : Indeed, $E\left(T_{d_{x} a_{y}}\right) \backslash F$ is the disjoint union of $E\left(T_{d_{z} a_{w}}\right) \backslash F^{\prime}$ and $\left\{d_{x} d_{z}, d_{x} a_{y}\right\}$.

- $(\Sigma, R)$ is "strong": The only inner-face of $T_{d_{x} a_{y}}$ that is not an inner-face of $T_{d_{z}} a_{w}$ is $d_{x} d_{z} a_{y}$, which corresponds to the new face-region $\mathbf{d}_{\mathbf{x}} \mathbf{d}_{\mathbf{z}} \mathbf{a}_{\mathbf{y}}$.

Finally we see in Fig. 21 that the other points of Property 2 are satisfied.

Case 2.2.3: $z=x$ and $w>y+1$ (see Fig. 22) Let us denote by $e_{1}, e_{2}, \ldots, e_{t}$ the neighbors of $d_{x}$ strictly inside the cycle $\left(d_{x}, a_{y}, \ldots, a_{w}, d_{x}\right)$, going "from right to left" (see Fig. 22). Since there is no chord $a_{i} a_{j}$, we have $t \geq 1$. Furthermore by minimality of $w$ we have $e_{i} \neq a_{j}$ for all $1 \leq i \leq t$ and $y \leq j \leq w$. Let $T_{1}$ be the subgraph of $T_{d_{x} a_{y}}$ that lies inside the cycle $\left(a_{y}, \ldots, a_{w}, e_{1}, \ldots, e_{t}, a_{y}\right)$. By Lemma 2, $T_{1}$ is a W-triangulation. Since the W-triangulation $T_{d_{x} a_{y}}$ has no separating 3-cycle $\left(d_{x}, a_{w}, e_{i}\right)$ or $\left(d_{x}, e_{i}, e_{j}\right)$, there exists no chord $a_{w} e_{i}$ or $e_{i} e_{j}$ in $T_{1}$. With the fact that 
Fig. 22 Case 2.2.3:

$T_{d_{x} a_{y}} \neq T_{d_{1} a_{p}}, z=x$, and $w>y+1$
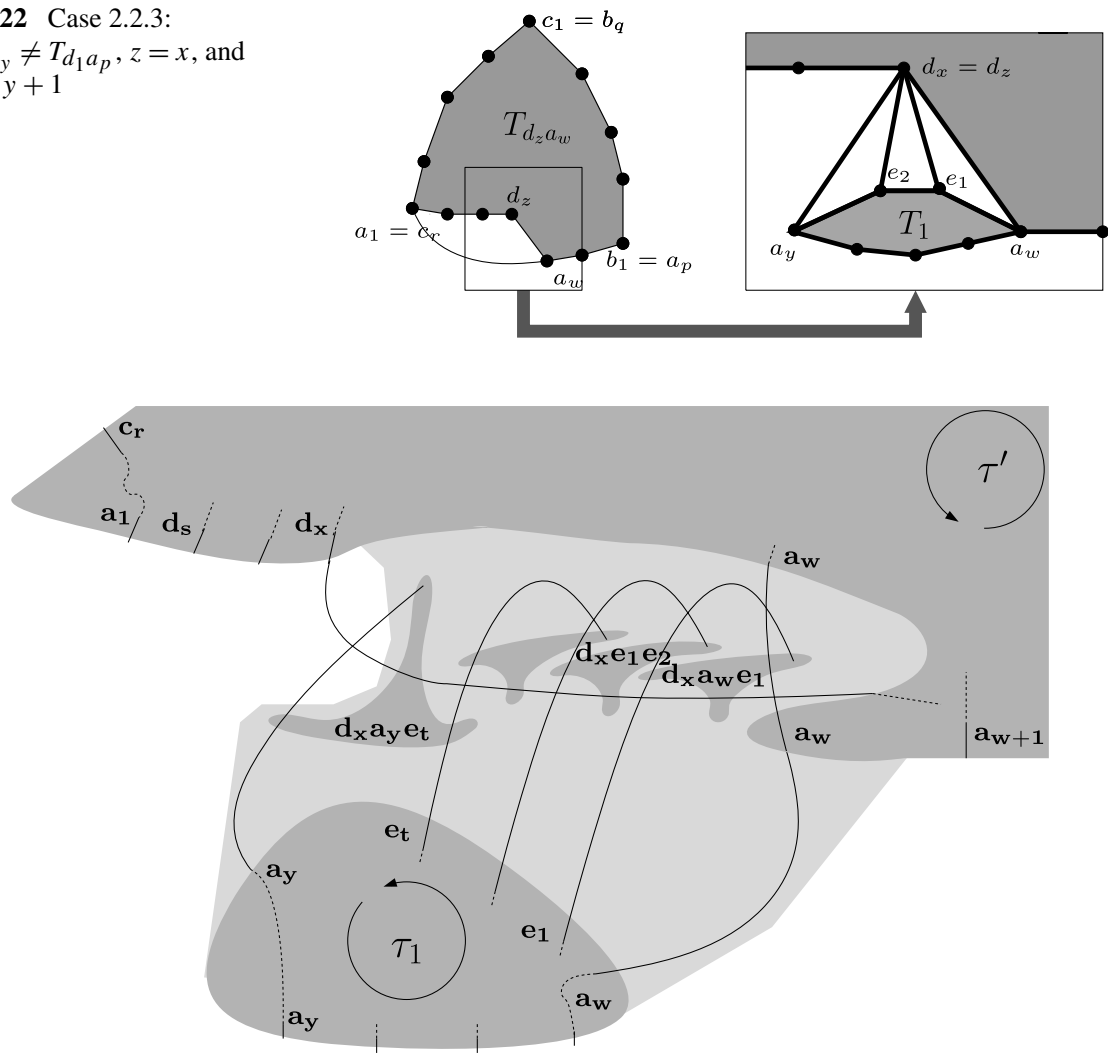

Fig. 23 Case 2.2.3: $(\Sigma, R, F)$

$t \geq 1$, we know that $\left(e_{t}, a_{y}\right)-\left(a_{y}, \ldots, a_{w}\right)-\left(a_{w}, e_{1}, \ldots, e_{t}\right)$ is a 3-boundary of $T_{1}$. Finally, since $T_{1}$ has less edges than $T_{d_{x} a_{y}}\left(d_{x} a_{y} \notin E\left(T_{1}\right)\right)$, Property 1 holds for $T_{1}$ with respect to the mentioned 3-boundary. Let $\left(\Sigma_{1}, R_{1}, F_{1}\right)$ be an anticlockwise PSrepresentation contained in the region $\tau_{1}$ obtained for $T_{1}$ with $F_{1}=E_{0}\left(T_{1}\right) \backslash\left\{e_{t} a_{y}\right\}$.

In Fig. 23, starting from $\left(\Sigma^{\prime}, R^{\prime}, F^{\prime}\right)$ and $\left(\Sigma_{1}, R_{1}, F_{1}\right)$, we show how to join the strings $\mathbf{a}_{\mathbf{w}}$ of $\Sigma^{\prime}$ and $\Sigma_{1}$, how to extend the string $\mathbf{a}_{\mathbf{y}}$ and the strings $\mathbf{e}_{\mathbf{i}}$ for $1 \leq i \leq t$, and how to draw the face-regions $\mathbf{a}_{\mathbf{y}} \mathbf{e}_{\mathbf{t}} \mathbf{d}_{\mathbf{x}}, \mathbf{e}_{\mathbf{1}} \mathbf{a}_{\mathbf{w}} \mathbf{d}_{\mathbf{x}}$, and $\mathbf{e}_{\mathbf{i}} \mathbf{e}_{\mathbf{i}-\mathbf{1}} \mathbf{d}_{\mathbf{x}}$ for $1<i \leq t$, in order to obtain $(\Sigma, R, F)$, an anticlockwise PS-representation of $T_{d_{x} a_{y}}$ contained in a region $\tau$.

We verify that $(\Sigma, R, F)$ satisfies Property 2 :

- $\Sigma$ is a 1-string representation of $T_{d_{x} a_{y}} \backslash F$ with $F=E_{\mathrm{o}}\left(T_{d_{x} a_{y}}\right) \backslash\left\{d_{x} a_{y}\right\}$ : Indeed, $E\left(T_{d_{x} a_{y}}\right) \backslash F$ is the disjoint union of $E\left(T_{d_{z} a_{w}}\right) \backslash F^{\prime}, E\left(T_{1}\right) \backslash F_{1}$, and $\left\{a_{w} e_{1}, d_{x} a_{y}\right\} \cup$ $\left\{e_{i} e_{i-1} \mid i \in[2, t]\right\} \cup\left\{d_{x} e_{i} \mid i \in[1, t]\right\}$.

- $(\Sigma, R)$ is "strong": The only inner-faces of $T_{d_{x} a_{y}}$ that are not inner-faces in $T_{d_{z} a_{w}}$ or $T_{1}$ are $d_{x} a_{y} e_{t}, d_{x} a_{w} e_{1}$, and the faces $d_{x} e_{i} e_{i-1}$ for $2 \leq i \leq t$, which correspond to the new face-regions.

Finally we see in Fig. 23 that the other points of Property 2 are satisfied. 

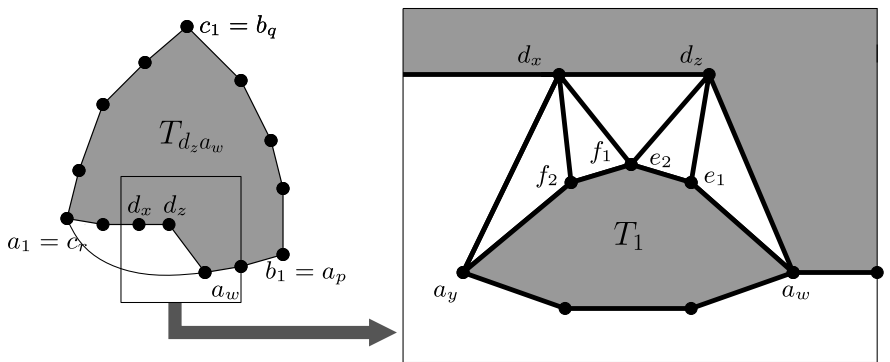

Fig. 24 Case 2.2.4: $T_{d_{x} a_{y}} \neq T_{d_{1} a_{p}}, z=x-1$, and $w>y$

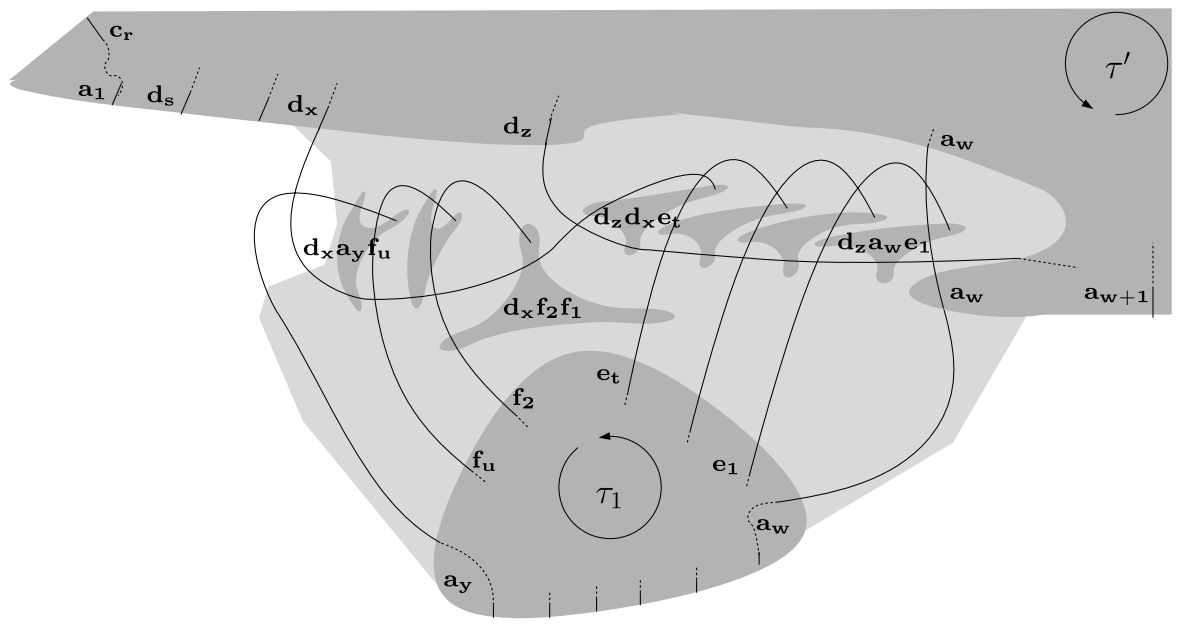

Fig. 25 Case 2.2.4: $(\Sigma, R, F)$

Case 2.2.4: $z=x-1$ and $w>y$ (see Fig. 24) Let us denote by $e_{1}, e_{2}, \ldots, e_{t}$ the neighbors of $d_{z}$ strictly inside the cycle $\left(d_{z}, d_{x}, a_{y}, \ldots, a_{w}, d_{z}\right)$, going "from right to left" (see Fig. 24). By maximality of $z$, there is no edge $d_{x} a_{w}$, so $t \geq 1$. Let us denote by $f_{1}, \ldots, f_{u}$ the neighbors of $d_{x}$ strictly inside the cycle $\left(d_{x}, a_{y}, \ldots, a_{w}, d_{z}, d_{x}\right)$, going "from right to left" (see Fig. 24). Note that $f_{1}=e_{t}$ and that by minimality of $w$, there is no edge $d_{z} a_{y}$, so $u \geq 1$.

By minimality of $w$ (resp. maximality of $z$ ) we have $e_{i} \neq a_{j}$ (resp. $f_{i} \neq a_{j}$ ) for all $1 \leq i \leq t$ (resp. $1 \leq i \leq u)$ and $y \leq j \leq w$. Let $T_{1}$ be the subgraph of $T_{d_{x} a_{y}}$ that lies inside the cycle $\left(a_{y}, \ldots, a_{w}, e_{1}, \ldots, e_{t}, f_{2}, \ldots, f_{u}, a_{y}\right)$. By Lemma $2, T_{1}$ is a Wtriangulation. Since the W-triangulation $T_{d_{x} a_{y}}$ has no separating 3-cycle $\left(d_{z}, a_{w}, e_{i}\right)$, $\left(d_{z}, e_{i}, e_{j}\right),\left(d_{x}, f_{i}, f_{j}\right)$, or $\left(d_{x}, f_{i}, a_{y}\right)$, there exists no chord $a_{w} e_{i}, e_{i} e_{j}, f_{i} f_{j}$, or $f_{i} a_{y}$ in $T_{1}$. With the fact that $t \geq 1$ and $u \geq 1$, we know that $\left(f_{1}, f_{2}, \ldots, f_{u}, a_{y}\right)-$ $\left(a_{y}, \ldots, a_{w}\right)-\left(a_{w}, e_{1}, \ldots, e_{t}\right)$ is a 3-boundary of $T_{1}$. Finally, since $T_{1}$ has less edges than $T_{d_{x} a_{y}}\left(d_{x} a_{y} \notin E\left(T_{1}\right)\right)$, Property 1 holds for $T_{1}$ with respect to the mentioned 3-boundary. Let $\left(\Sigma_{1}, R_{1}, F_{1}\right)$ be an anticlockwise PS-representation contained in the region $\tau_{1}$ obtained for $T_{1}$ with $F_{1}=E_{0}\left(T_{1}\right) \backslash\left\{f_{1} f_{2}\right\}$. 
Fig. 26 S-representation of $T$ from $(\Sigma, R, F)$

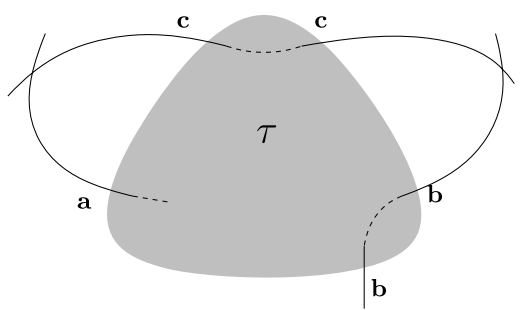

In Fig. 25, starting from $\left(\Sigma^{\prime}, R^{\prime}, F^{\prime}\right)$ and $\left(\Sigma_{1}, R_{1}, F_{1}\right)$, we show how to join the strings $\mathbf{a}_{\mathbf{w}}$ of $\Sigma^{\prime}$ and $\Sigma_{1}$, how to extend the string $\mathbf{d}_{\mathbf{x}}, \mathbf{a}_{\mathbf{y}}$, the strings $\mathbf{e}_{\mathbf{i}}$ for $1 \leq i \leq t$, and the strings $\mathbf{f}_{\mathbf{i}}$ for $2 \leq i \leq u$, and how to draw the face-regions $\mathbf{d}_{\mathbf{z}} \mathbf{a}_{\mathbf{w}} \mathbf{e}_{1}, \mathbf{d}_{\mathbf{z}} \mathbf{e}_{\mathbf{i}} \mathbf{e}_{\mathbf{i}-\mathbf{1}}$ for $2 \leq i \leq t, \mathbf{d}_{\mathbf{z}} \mathbf{d}_{\mathbf{x}} \mathbf{e}_{\mathbf{t}}, \mathbf{d}_{\mathbf{x}} \mathbf{f}_{\mathbf{i}} \mathbf{f}_{\mathbf{i}-\mathbf{1}}$ for $2 \leq i \leq u$, and $\mathbf{d}_{\mathbf{x}} \mathbf{a}_{\mathbf{y}} \mathbf{f}_{\mathbf{u}}$ in order to obtain $(\Sigma, R, F)$, an anticlockwise PS-representation of $T_{d_{x} a_{y}}$ almost contained in a region $\tau$.

We verify that $(\Sigma, R, F)$ satisfies Property 2 :

- $\Sigma$ is a 1-string representation of $T_{d_{x} a_{y}} \backslash F$ with $F=E_{\mathrm{o}}\left(T_{d_{x} a_{y}}\right) \backslash\left\{d_{x} a_{y}\right\}$ : Indeed, $E\left(T_{d_{x} a_{y}}\right) \backslash F$ is the disjoint union of $E\left(T_{d_{z} a_{w}}\right) \backslash F^{\prime}, E\left(T_{1}\right) \backslash F_{1}$, and $\left\{d_{x} a_{y}, d_{x} d_{z}, a_{w} e_{1}, a_{y} f_{u}\right\} \cup\left\{d_{z} e_{i} \mid i \in[1, t]\right\} \cup\left\{d_{x} f_{i} \mid i \in[1, u]\right\} \cup\left\{e_{i} e_{i-1} \mid i \in\right.$ $[2, t]\} \cup\left\{f_{i} f_{i-1} \mid i \in[2, u]\right\}$.

- $(\Sigma, R)$ is "strong": The only inner-faces of $T_{d_{x} a_{y}}$ that are not inner-faces in $T_{d_{z} a_{w}}$ or $T_{1}$ are $d_{z} a_{w} e_{1}, d_{z} e_{i} e_{i-1}$ for $2 \leq i \leq t, d_{z} d_{x} e_{t}, d_{x} f_{i} f_{i-1}$ for $2 \leq i \leq u$, and $d_{x} a_{y} f_{u}$, which correspond to the new face-regions.

Finally we see in Fig. 25 that the other points of Property 2 are satisfied. So, Property 2 holds for any W-triangulation $T_{d_{x} a_{y}}$ with $m$ edges, and this completes the proofs of Properties 1 and 2.

\section{Proof in the General Case}

Theorem 3 Every triangulation $T$ admits an $S$-representation $(\Sigma, R)$.

Proof We prove this result by induction on the number of separating 3-cycles. Note that any triangulation $T$ is 3-connected and that if $T$ has no separating 3-cycle, then $T$ is 4-connected and is a W-triangulation. Consequently, if $T$ is a 4-connected triangulation whose outer-vertices are $a, b$, and $c$, then $T$ is a W-triangulation 3-bounded by $(a, b)-(b, c)-(c, a)$. By Property $1, T$ admits a PS-representation $(\Sigma, R, F)$, with $F=\{b c, c a\}$, that is contained in a region $\tau$. Furthermore, in the boundary of $\tau$, we successively meet the ends of $\mathbf{b}, \mathbf{b}, \mathbf{c}, \mathbf{c}, \mathbf{a}$. To obtain an S-representation of $T$, it is sufficient to extend $\mathbf{a}, \mathbf{b}$, and $\mathbf{c}$ outside of $\tau$ so that $\mathbf{c}$ crosses $\mathbf{a}$ and $\mathbf{b}$, as depicted in Fig. 26.

Suppose now that $T$ is a triangulation that contains at least one separating 3-cycle. Consider a separating 3-cycle $(a, b, c)$ such that there is no other separating 3-cycle lying inside. This implies that the triangulation $T^{\prime}$ induced by the vertices on and inside $(a, b, c)$ is 4-connected.

Let $T_{1}$ be the triangulation obtained by removing the vertices lying strictly inside $(a, b, c)$. Let $T_{2}$ be the subgraph of $T$ induced by the vertices lying strictly inside 
Fig. 27 In the S-representation $\left(\Sigma_{1}, R_{1}\right)$ of $T_{1}$, the $(a, b, c)$-region abc

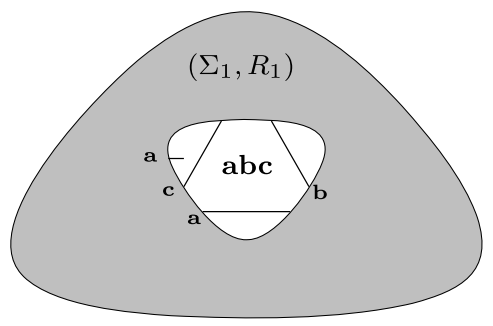

Fig. 28 The cases (A) and (B)
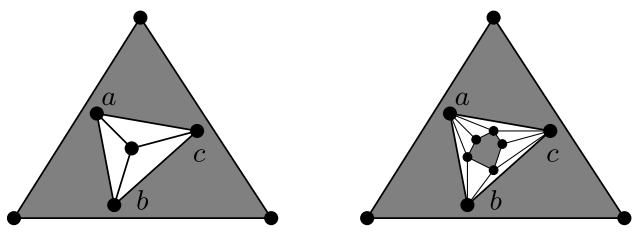

$(a, b, c)$ (i.e., $\left.T_{2}=T^{\prime} \backslash\{a, b, c\}\right)$. In $T_{1}$, the cycle $(a, b, c)$ is a face of the triangulation and is no more a separating 3 -cycle. Thus, $T_{1}$ has one separating cycle less than $T$, and so we have by induction hypothesis that $T_{1}$ admits an S-representation $\left(\Sigma_{1}, R_{1}\right)$. This S-representation contains a face-region abc corresponding to the face $a b c$. Without loss of generality, say that abc is an $(a, b, c)$-region, as depicted in Fig. 27.

Since $T^{\prime}$ is a triangulation with at least four vertices, the neighbors of any vertex $v \in V\left(T^{\prime}\right)$ induce a cycle. Suppose that the vertex $a$ (resp. $b$ and $c$ ) has exactly one neighbor $v$ that lies inside $(a, b, c)$. Then there exists a cycle $(b, v, c)(\operatorname{resp} .(a, v, c)$ and $(a, v, b))$ in $T^{\prime}$, and consequently $v$ is a neighbor of $a, b$, and $c$ in $T^{\prime}$. Suppose that there exists another vertex $w$ in $T^{\prime}$, then $w$ lies either inside the cycle $(a, v, b)$, inside $(a, v, c)$, or inside $(b, v, c)$, and then one of these cycles is a separating 3cycle. This is impossible by definition of $(a, b, c)$. So we can distinguish two cases (see Fig. 28), (A) the case where $T_{2}$ is a single vertex, and (B) the case where each of the vertices $a, b$, and $c$ has at least two neighbors inside $(a, b, c)$.

Case (A): $T_{2}$ is a single vertex $v$ To obtain an S-representation $(\Sigma, R)$ of $T$ (see Fig. 29), we add a string $\mathbf{v}$ in $\left(\Sigma_{1}, R_{1}\right)$. Since $E(T) \backslash E\left(T_{1}\right)=\{v a, v b, v c\}$, this string $\mathbf{v}$ crosses $\mathbf{a}, \mathbf{b}, \mathbf{c}$. Moreover, we also define three disjoint face-regions acv, vbc, vab that correspond respectively to the faces $a c v, v b c, v a b$.

Since $\left(\Sigma_{1}, R_{1}\right)$ is an S-representation of $T_{1}$ and since $\mathbf{v}, \mathbf{a c v}, \mathbf{v b c}, \mathbf{v a b}$ are drawn inside abc, it is clear that $(\Sigma \cup\{\mathbf{v}\},(R \backslash\{\mathbf{a b c}\}) \cup\{\mathbf{a c v}, \mathbf{v b c}, \mathbf{v a b}\})$ is an $\mathrm{S}$ representation of $T$.

Case (B): Each of the vertices $a, b$, and c has at least two neighbors inside $(a, b, c)$ There exists a cycle $\left(c, a_{1}, \ldots, a_{p}, b\right)\left(\operatorname{resp} .\left(a, b_{1}, \ldots, b_{q}, c\right)\right.$ and $\left.\left(b, c_{1}, \ldots, c_{r}, a\right)\right)$ in $T^{\prime}$ whose vertices are exactly the neighbors of $a$ (resp. $b$ and $c$ ). We already know that $p>1, q>1$, and $r>1$ and that $a_{p}=b_{1}, b_{q}=c_{1}$, and $c_{r}=a_{1}$. Moreover, since $b_{1}$ and $c$ (resp. $c_{1}$ and $a$, and $a_{1}$ and $b$ ) are the only two common neighbors of $a$ and $b$ (resp. $b$ and $c$, and $a$ and $c$ ) in $T^{\prime}$ (otherwise there would be a separating 3-cycle), 
Fig. 29 Case (A):

Modifications inside abc

Fig. 30 Case (B)

Modifications inside abc
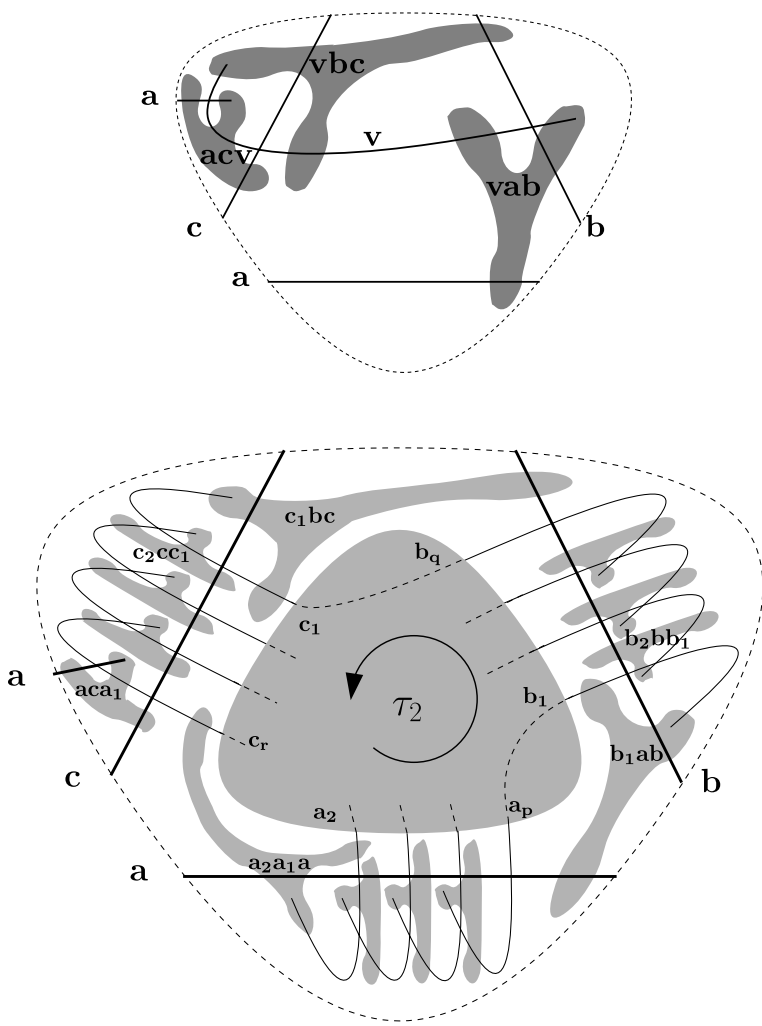

we have that $\left(a_{1}, \ldots, a_{p}=b_{1}, \ldots, b_{q}=c_{1}, \ldots, c_{r}=a_{1}\right)$ is a cycle. This implies by Lemma 2 that $T_{2}$ is a $\mathrm{W}$-triangulation.

Suppose that there exists an edge $a_{i} a_{j}$ (resp. $b_{i} b_{j}, c_{i} c_{j}$ ) with $1<i+1<j \leq$ $p$ (resp. $1<i+1<j \leq q, 1<i+1<j \leq r$ ). Then, the cycle $\left(a, a_{i}, a_{j}\right)$ (resp. $\left.\left(b, b_{i}, b_{j}\right),\left(c, c_{i}, c_{j}\right)\right)$ would be a separating 3-cycle of $T^{\prime}$. Consequently, $T_{2}$ is 3bounded by $\left(a_{1}, \ldots, a_{p}\right)-\left(b_{1}, \ldots, b_{q}\right)-\left(c_{1}, \ldots, c_{r}\right)$. With respect to this 3-boundary, $T_{2}$ has an anticlockwise PS-representation $\left(\Sigma_{2}, R_{2}, F_{2}\right)$ with $F_{2}=E_{\mathrm{o}} \backslash\left\{a_{1} a_{2}\right\}$ (cf. Property 1). Let $\tau_{2}$ be a region of abc containing this representation.

Since abc is an $(a, b, c)$-region, on its boundary we successively cross $\mathbf{a}, \mathbf{a}, \mathbf{b}, \mathbf{b}, \mathbf{c}$, $\mathbf{a}$, and $\mathbf{c}$ when going anticlockwise (by doing an axial symmetry if necessary).

In Fig. 30, starting from $\left(\Sigma_{1}, R_{1}\right)$ and $\left(\Sigma_{2}, R_{2}\right)$, we obtain $(\Sigma, R)$. We extend the strings $\mathbf{a}_{2}, \ldots, \mathbf{a}_{\mathbf{p}}, \mathbf{b}_{\mathbf{1}}, \ldots, \mathbf{b}_{\mathbf{q}}, \mathbf{c}_{\mathbf{1}}, \ldots, \mathbf{c}_{\mathbf{r}}$ to obtain the crossings that correspond to the edges in the set $E(T) \backslash\left(E\left(T_{1}\right) \cup\left(E\left(T_{2}\right) \backslash F_{2}\right)\right)=\left\{a a_{i} \mid i \in[1, p]\right\} \cup\left\{b b_{i} \mid i \in[1, q]\right\} \cup$ $\left\{c c_{i} \mid i \in[1, r]\right\} \cup\left\{a_{i} a_{i+1} \mid i \in[2, p-1]\right\} \cup\left\{b_{i} b_{i+1} \mid i \in[1, q-1]\right\} \cup\left\{c_{i} c_{i+1} \mid i \in\right.$ $[1, r-1]\}$. We also define face-regions for the faces in the set $\left\{a b b_{1}, a c a_{1}, b c c_{1}\right\} \cup$ $\left\{a a_{i} a_{i+1} \mid i \in[1, p-1]\right\} \cup\left\{b b_{i} b_{i+1} \mid i \in[1, q-1]\right\} \cup\left\{c c_{i} c_{i+1} \mid i \in[1, r-1]\right\}$.

Since $\left(\Sigma_{1}, R_{1}\right)$ is an S-representation of $T_{1}$ and $\left(\Sigma_{2}, R_{2}, F_{2}\right)$ is a PS-representation of $T_{2},(\Sigma, R, F)$ is an S-representation of $T$.

- $\Sigma$ is a 1-string representation of $T$ : Indeed, we added all the crossings corresponding to the edges in $E(T) \backslash\left(E\left(T_{1}\right) \cup\left(E\left(T_{2}\right) \backslash F_{2}\right)\right)$. 
- $(\Sigma, R)$ is "strong": Indeed, we added all the face-regions corresponding to the inner-faces of $T$ that are neither in $T_{1}$ nor in $T_{2}$.

Consequently, every triangulation admits an S-representation, which proves Theorem 3 and then Theorem 2.

\section{Conclusion}

The first and the second author recently improved the result presented in this article by proving Conjecture 1 [2]. For this, they use the same decomposition of triangulation, but their notion of face-region is quite different. One should also mention that their construction does not correspond to a stretching of the 1-string representation presented here.

Finally, an interesting question is whether the result presented here holds for other surfaces. For example, does any graph embedded on a surface $\mathbb{S}$ have a 1-string representation on $\mathbb{S}$ ?

\section{References}

1. de Castro, N., Cobos, F., Dana, J.C., Márquez, A., Noy, M.: Triangle-free planar graphs as segment intersection graphs. J. Graph Algorithms Appl. 6(1), 7-26 (2002)

2. Chalopin, J., Gonçalves, D.: Every planar graph is the intersection graph of segments in the plane. In: Proceedings of the 41st Annual ACM Symposium on Theory of Computing (2009)

3. Ehrlich, G., Even, S., Tarjan, R.E.: Intersection graphs of curves in the plane. J. Combin. Theory., Ser. B 21, 8-20 (1976)

4. de Fraysseix, H., Ossona de Mendez, P., Pach, J.: Representation of planar graphs by segments. Intuit. Geom. (Szeged, 1991), Colloq. Math. Soc. János Bolyai 63, 109-117 (1994)

5. de Fraysseix, H., Ossona de Mendez, P.: Intersection graphs of Jordan arcs. DIMACS Ser. Discrete Math. Theor. Comput. Sci. 49, 11-28 (1999)

6. de Fraysseix, H., Ossona de Mendez, P.: Representations by contact and intersection of segments. Algorithmica 47(4), 453-463 (2007)

7. Gonçalves, D.: Edge-partition of planar graphs into two outerplanar graphs. In: Proceedings of the 37th Annual ACM Symposium on Theory of Computing, pp. 504-512 (2005)

8. Hartman, I.B.-A., Newman, I., Ziv, R.: On grid intersection graphs. Discrete Math. 87(1), 41-52 (1991)

9. Koebe, P.: Kontaktprobleme der Konformen Abbildung. Ber. Sächs. Akad. Wiss. Leipzig, Math.Phys. K1. 88, 141-164 (1936)

10. Scheinerman, E.R.: Intersection classes and multiple intersection parameters of graphs. PhD Thesis, Princeton University (1984)

11. West, D.: Open problems. SIAM J. Discrete Math. Newslett. 2(1), 10-12 (1991)

12. Whitney, H.: A theorem on graphs. Ann. Math. (2) 32(2), 378-390 (1931) 